\title{
A logic for concepts and similarity
}

\author{
M. Sheremet, D. Tishkovsky, F. Wolter, and M. Zakharyaschev ${ }^{1}$ \\ ${ }^{1}$ School of Computer Science and \\ Information Systems, Birkbeck College \\ Malet Street, London WC1E 7HX, U.K. \\ \{mikhail,michael\}@dcs.bbk.ac.uk \\ ${ }^{2}$ Department of Computer Science \\ University of Liverpool \\ Liverpool L69 3BX, U.K. \\ \{dmitry,frank\}@csc.liv.ac.uk
}

\begin{abstract}
Categorisation of objects into classes is currently supported by (at least) two 'orthogonal' methods. In logic-based approaches, classifications are defined through ontologies or knowledge bases which describe the existing relationships among terms. Description logic (DL) has become one of the most successful formalisms for representing such knowledge bases, in particular because theoretically well-founded and efficient reasoning tools have been readily available.

In numerical approaches, classifications are obtained by first computing similarity (or proximity) measures between objects and then categorising them into classes by means of Voronoi tessellations, clustering algorithms, nearest neighbour computations, etc.

In many areas such as bioinformatics, computational linguistics or medical informatics, these two methods have been used independently of each other: although both of them are often applied to the same domain (and even by the same researcher), up to now no formal interaction mechanism has been developed.

In this paper, we propose a DL-based integration of the two classification methods. Our formalism, called $\mathcal{S L}+\mathcal{A L C} \mathcal{L I O}$, extends the expressive DL $\mathcal{A L C} \mathcal{Q} \mathcal{I O}$ by means of the constructors of the similarity logic $\mathcal{S} \mathcal{L}$ which allow definitions of concepts in terms of both comparative and absolute similarity. In the combined knowledge base the user should declare the similarity spaces where the new operators are interpreted.

Of course, $\mathcal{S} \mathcal{L}+\mathcal{A L C} \mathcal{Q I O}$ can only be useful if classifications with this logic are supported by automated reasoning tools. We lay theoretical foundations for the development of such tools by showing that reasoning problems for $\mathcal{S L}+\mathcal{A L C Q \mathcal { O }}$ can be decomposed into the corresponding problems for its DL-part $\mathcal{A L C} \mathcal{L I O}$ and similarity part $\mathcal{S L}$. Then we investigate reasoning in $\mathcal{S} \mathcal{L}$ and prove that consistency and many other reasoning problems are ExpTime-complete for this logic. Using this result and a recent complexity result of Pratt-Hartmann for $\mathcal{A L C Q \mathcal { O }}$, we prove that reasoning in $\mathcal{S L}+\mathcal{A L C Q \mathcal { O }}$ is NExpTime-complete.

As the 'closer' operator of $\mathcal{S} \mathcal{L}$ has the same expressive power as the standard implication $>$ of conditional logic, these results may have interesting consequences for conditional logic as well.
\end{abstract}

Keywords: similarity logic, description logic, ontology, complexity, conditional logic.

\section{Introduction}

Classification — "the actual or ideal arrangement together of those which are like, and the separation of those which are unlike" (see $[28,23]$ and references therein) — is said to be one of 
the main objectives of science, the Linnaean taxonomy and the Mendeleev periodic table being probably the best known witnesses. Modern classification and conceptualisation problems in bioinformatics, linguistics or the World Wide Web are characterised by huge numbers of objects to be categorised and/or their very complex structures. Building adequate 'ontologies' for such areas and developing powerful tools for dealing with them have been recognised among the most important and urgent tasks, and can be regarded as a real challenge for the knowledge representation and reasoning (KR\&R) community.

There are (at least) three general ways of categorising things into classes:

- by establishing logical relationships between them (e.g., 'a mother is a woman with a child'),

- by identifying prototypical (the most representative) instances of classes against which the surrounding 'poorer' instances are categorised (e.g., a colour is blueish if it is more similar to the prototypical colour blue than to the prototypical colours green, red, etc.),

- by means of clustering, that is, by partitioning a set of objects into subsets (clusters), so that the objects in each subset are more similar to each other than to the objects in other subsets.

From the KR point of view, the main difference between these approaches is that the first one is logic-based and usually involves building knowledge bases or ontologies in such formalisms as description logic or (fragments of) first-order logic, while the last two are 'quantitative' in that they are based on implicit or explicit similarity (or proximity) measures and use numerical computations (say, Voronoi tessellations, nearest neighbour or clustering algorithms). Successful examples of the logic-based approach are such well-known ontologies in medicine and bioinformatics as SNOMED (www.snomed.org) or GO (www.geneontology.org). On the other hand, in those (still huge) areas of bioinformatics, computational linguistics, etc. where there exist vast amounts of poorly investigated and unstructured data, the quantitative, similarity-based methods (say, using alignment algorithms and tools like BLAST [1]) often seem to be the only choice.

The apparent 'qualitative-quantitative orthogonality' of these approaches was probably the reason why they have not been formally integrated - despite the fact that researchers in bioinformatics and linguistics as well as 'ordinary people' in their everyday life use all these methods frequently and in parallel (see, e.g., [40]). We will illustrate this point by two simple examples from the 'World of Whisky.' The classification at www . whiskymag. com can easily be represented in a sufficiently expressive description logic. For instance, the definition 'vatted malt Speyside whisky is malt whisky blended from several distilleries located in the Speyside region of Scotland' can be formalised as

$$
\begin{aligned}
& \text { Vatted_malt_Speyside_whisky } \equiv \\
& \qquad \text { Malt_whisky } \sqcap \geq 2 \text { made_by.Distillery } \sqcap \forall \text { made_by.Jis_located_in.\{Speyside\} }
\end{aligned}
$$

One would hardly like to replace this clear axiom with a quantitative similarity-based representation. In general, we do not see any evidence that ontologies could be built purely on the basis of, say, conceptual similarity spaces in the sense of [10].

On the other hand, it appears to be extremely difficult to define by means of logical relations the taste characteristics of whiskies in terms of natural factors such as the geographical region, the type of rock from which the water source originates, the amount of peat, etc. 
For example, the classification of malt whiskies according to their taste in [37] is based on computing a proximity matrix and does not correspond to more traditional regional (and other) classifications ('the regional classification is really only helpful today if you are trying to visit a distillery'). Again, there appears to be no evidence that logic-based formalisms could replace such similarity-based classifications.

Given that both approaches are applied for classifying objects from the same domain (and, moreover, can potentially correct errors of each other ${ }^{1}$ ), it seems natural to try to integrate them into a single knowledge representation formalism and support it with suitable reasoning and classification tools.

In this paper we propose an integration of logic-based and similarity-based approaches of the following form. A knowledge base consists of three ingredients: a set of axioms fixing the terminology (usually called a TBox), a set of assertions about individuals (an ABox), and a declaration of similarity spaces that are used in the TBox and ABox. The TBox defines logical relations between concepts as it is by now standard in description logics (DLs); see the example above. However, in contrast to standard DLs, we do not only consider definitions within abstract spaces of concepts, but also those based on similarity measures (these similarity measures should not be confused with semantic similarity considered, e.g., in [27]). First, we allow definitions by means of 'comparative similarity.' To be able to use comparative similarity in concept definitions, the user has to declare a similarity space by means of an atomic concept comprising the elements of the space and certain constraints on the space (saying that it is symmetric and/or satisfies the triangle inequality; for a discussion of these properties of similarity see, e.g., [35]). For example, in the World of Whisky, one could declare a similarity space for comparing 'tastes' by introducing a concept name Taste which comprises the set of all elements of the (possibly not completely specified) similarity space with a similarity measure $d_{\text {Taste }}$ on it. Using the role has_taste and its inverse taste_of, the ontology designer can first introduce the taste of a whisky (e.g., of Caol Ila) by the axiom

$$
\text { T_Caol_lla } \equiv \text { Taste } \sqcap \exists \text { taste_of. }\{\text { Caol_lla }\}
$$

and then define, for example,

Peaty_malt $\equiv$ Single_malt $\sqcap$ Ghas_taste.(T_Caol_Ila $\leftleftarrows$ Taste $T$ _Macallan $\sqcup \cdots \sqcup$ T_Glenmorangie).

Intuitively, this TBox axiom states that peaty malt whiskies are those single malt whiskies that are more similar regarding their taste to (the prototype) Caol Ila than to (the prototypes) Macallan, ..., Glenmorangie. Notice that, as Taste is a concept, we can further refine the notion of taste in the TBox by means of various flavour and taste descriptors: for example,

$$
\text { Taste } \sqsubseteq \text { ᄏhas_taste_descriptor. (Winey } \sqcup \text { Woody } \sqcup \cdots \sqcup \text { Peaty). }
$$

Along with comparative similarity, the user has an option to define concepts in terms of 'absolute similarity' which can, for example, be found using numerical techniques in the proximity matrix:

$$
\text { T_Caol_lla } \sqsubseteq \neg \exists<\exists_{\text {Taste }}^{<.67} \exists \text { taste_of. Medium_sweet_whisky }
$$

says that the taste of Caol Ila cannot be too close (at most 0.67 close) to the taste of medium sweet whiskies.

\footnotetext{
${ }^{1}$ For example, the clustering algorithm in [19] places the Hungarian language into the same family as Turkish, which contradicts the existing ontologies.
} 
Besides the TBox, the knowledge base contains an ABox which provides data about concrete objects. First, one can specify within description logic:

$$
\text { Lagavulin : Dry_whisky_ Lagavulin_Distillery is_located_in Islay }
$$

where the former says that Lagavulin is a dry whisky. Second, one can include information about similarities between concrete objects by writing down (part of the) proximity matrix in assertions of the form

$$
d_{\text {Taste }}\left(\ell_{1}, \ell_{2}\right)=0.15 \quad d_{\text {Taste }}\left(\ell_{3}, \ell_{4}\right)<1
$$

The resulting knowledge representation formalism, called $\mathcal{S L}+\mathcal{A L C} \mathcal{Q I O}$, is a combination of two logics. One of them is a suitable DL (see [2] for a survey); in this paper we consider the expressive DL $\mathcal{A L C} \mathcal{Q I O}$, but it could be replaced with any other 'well-behaved' DL. The second one is the similarity logic $\mathcal{S} \mathcal{L}$, which itself is a combination of two logics:

- the logic $\mathcal{M S}$ of 'absolute' similarity with concept constructors of the form $\exists^{<a} C$ and $\exists \leq a C$, that is, 'in the $a$-neighbourhood of $C$ ' (excluding or including the boundary), where $a$ is a positive rational number (this and other similar logics were first introduced and investigated in $[17,38,22,39])$, and

- the logic $\mathcal{C S L}$ of 'comparative' similarity with the 'closer' operator $C \leftleftarrows D$ which is interpreted by the set of all points in the similarity space that are closer to the instances of $C$ than to the instances of $D$. For example, it can be used to model statements like ' $X$ resembles $C$ more than $D$.' This logic was first introduced in [30]. $\mathcal{C S} \mathcal{L}$ is closely related to conditional logics $[18,29]$; we will briefly discuss this connection in Section 5.

The applications of $\mathcal{S L}+\mathcal{A L C} \mathcal{Q I O}$ we envisage are similar to the use of DL in the process of ontology formation and maintenance, which means that reasoning with the logic is of fundamental importance (to check whether the resulting classification is consistent, whether it reflects properly the available knowledge, etc.); see $[15,24,14]$ for the pure DL-reasoners Fact, Pellet, and Racer.

The main contributions of this paper are as follows:

1. An analysis of the computational complexity of reasoning in the similarity logics $\mathcal{S} \mathcal{L}$ and $\mathcal{C S} \mathcal{L}$ interpreted in various distance spaces (in particular, metric spaces) satisfying the following application motivated min-condition: the distance between any two sets is the minimum, rather than infimum, of the distances between their points. We show that the satisfiability (or consistency) problem for either logic is ExpTime-complete over standard distance spaces with min-condition, even under the binary coding of the parameters in absolute distance operators of the logic $\mathcal{S} \mathcal{L}$. We also prove the finite model property of the logics under consideration. The ExpTime upper bound proofs depend heavily on whether the underlying distance spaces are assumed to be symmetric or not: in the non-symmetric case the upper bound is established by an exponential reduction to the emptiness problem for finite looping tree automata, whereas in the symmetric case it is proved by an exponential reduction to the emptiness problem for single complemented pair automata. (Note that the complexity of the logic $\mathcal{M S}$ of 'absolute' similarity has been determined in [39].)

2. A modular reasoning procedure for the combination $\mathcal{S L}+\mathcal{A L C} \mathcal{Q I O}$ of $\mathcal{S} \mathcal{L}$ and $\mathcal{A L C Q \mathcal { O }}$ is developed. Reasoning in this logic is shown to be NExpTime-complete. 


\section{Similarity description logic}

We begin by introducing the syntax and semantics of our logic. The language can be based on any of the standard DLs with the nominal constructor, say, the very expressive DL $\mathcal{S H O I} \mathcal{Q}$ [16] which is the core of the ontology language OWL-DL. For simplicity, in this paper we consider the DL $\mathcal{A L C} \mathcal{Q I O}$, that is, $\mathcal{S H O I} \mathcal{Q}$ without transitive roles and role hierarchies [2].

Language. Let $\mathrm{N}_{\mathrm{C}}$ be a countable set of concept names, $\mathrm{N}_{\mathrm{R}}$ a countable set of role names, $\mathrm{N}_{\mathrm{O}}$ a countable set of object names, and $\mathrm{S}$ a finite set of names for similarity spaces. The set $\mathrm{R}$ of roles is $\mathrm{N}_{\mathrm{R}} \cup\left\{R^{-1} \mid R \in \mathrm{N}_{\mathrm{R}}\right\}$.

The concepts of the 'similarity + description' language $\mathcal{S L}+\mathcal{A L C Q \mathcal { O }}$ are defined inductively as follows:

$$
\begin{aligned}
& C, D \quad:=A \quad|\quad\{\ell\} \quad| \quad C \sqcap D \quad|\quad \neg C| \leq n R . C \mid \geq n R . C \\
& C \leftleftarrows{ }_{s} D \quad\left|\quad \exists_{s}^{<a} C \quad\right| \quad \exists_{s}^{\leq a} C
\end{aligned}
$$

where $A$ ranges over $\mathrm{N}_{\mathrm{C}}, R$ over $\mathrm{R}, \ell$ over $\mathrm{N}_{\mathrm{O}}, n$ over the natural numbers $\mathbb{N}, a$ over the positive rational numbers $\mathbb{Q}^{>0}$, and $s$ over the names for similarity spaces from $\mathrm{S}$. As usual, $\exists R . C$ stands for $\geq 1 R$.C. We also use $\sqcup, \perp$ (for $\emptyset$ ), and $\top$ (for the whole space) as the standard Boolean abbreviations.

A knowledge base (KB, for short) in this language is a triple of the form $\Sigma=\left(\mathcal{T}^{\Sigma}, \mathcal{A}^{\Sigma}, \mathcal{D}^{\Sigma}\right)$, where

- $\mathcal{T}^{\Sigma}$ is a finite set (called a TBox) of subsumption relations $C \sqsubseteq D$ between concepts $C$ and $D$ of $\mathcal{S L}+\mathcal{A L C Q \mathcal { I O }}$

- $\mathcal{A}^{\Sigma}$ is a finite set (called an $A B o x$ ) of assertions of the form

$$
\ell_{1}: C, \quad \ell_{1} R \ell_{2}, \quad d_{s}\left(\ell_{1}, \ell_{2}\right)<a, \quad d_{s}\left(\ell_{1}, \ell_{2}\right)=a,
$$

where $C$ is a concept, $R$ a role, $s \in \mathrm{S}$, the $\ell_{i}$ are object names, $d_{s}$ is the distance (or similarity) measure on the distance (similarity) space $s$ (see below), and $a \in \mathbb{Q}^{>0}$,

- $\mathcal{D}^{\Sigma}$ associates with each $s \in \mathrm{S}$ a pair $\left(\mathcal{D}_{1}(s), \mathcal{D}_{2}(s)\right)$, where $\mathcal{D}_{1}(s)$ is a concept name from $\mathrm{N}_{\mathrm{C}}$ and $\mathcal{D}_{2}(s) \subseteq\{$ sym, tr $\}$ (to indicate whether the similarity space on $\mathcal{D}_{1}(s)$ is symmetric and/or satisfies the triangle inequality; see below for definitions).

As usual in DL, $C \equiv D$ will be used as an abbreviation for two subsumption axioms $C \sqsubseteq D$ and $C \sqsupseteq D$.

Models. To introduce models interpreting such knowledge bases, we need a definition of a distance (or similarity) space. Strictly speaking, the notions of distance and similarity are dual, but it will be more convenient for us not to distinguish between them here.

A distance space is a structure of the form $\mathfrak{D}=(D, d)$, where $D$ is a nonempty set and $d$, the distance function (or similarity measure), is a map from $D \times D$ to the set $\mathbb{R} \geq 0$ of nonnegative real numbers such that, for all $x, y \in D$, we have $d(x, y)=0$ iff $x=y$. If $d$ satisfies two additional well-known properties

$$
\begin{aligned}
& d(x, y)=d(y, x) \\
& d(x, z) \leq d(x, y)+d(y, z)
\end{aligned}
$$


then $\mathfrak{D}$ is a standard metric space. The distance $d(X, Y)$ between two nonempty sets $X$ and $Y$ of $D$ is defined by

$$
d(X, Y)=\inf \{d(x, y) \mid x \in X, y \in Y\}
$$

If at least one of $X, Y$ is empty then $d(X, Y)=\infty$. Finally, if we actually have

$$
d(X, Y)=\min \{d(x, y) \mid x \in X, y \in Y\},
$$

$(\min )$

for any nonempty $X, Y \subseteq D$, then $\mathfrak{D}$ is called a min-space. Every finite distance space is obviously a min-space. From now on we will only work with min-spaces. ${ }^{2}$ This means, in particular, that the topology induced by the distance spaces under consideration is trivial: every set of the space is both closed and open. Such a restriction seems to be quite natural for the intended applications, since most similarity spaces are either finite or can be approximated by min-spaces (unlike those in spatial and temporal KR\&R). It is of interest to observe that a similar restriction is required in the semantics of conditional logics (where it is called the limit assumption). The condition ( $\mathrm{min}$ ) and the relation between the logics considered here and conditional logics will be discussed in more detail in Section 5 .

Now, a $\Sigma$-type model (or interpretation) $\mathcal{I}$ consists of a nonempty domain $\Delta^{\mathcal{I}}$ and interpretations

- $\ell^{\mathcal{I}} \in \Delta^{\mathcal{I}}$, for each $\ell \in \mathrm{N}_{\mathrm{O}}$,

- $A^{\mathcal{I}} \subseteq \Delta^{\mathcal{I}}$, for each $A \in \mathrm{N}_{\mathrm{C}}$,

- $R^{\mathcal{I}} \subseteq \Delta^{\mathcal{I}} \times \Delta^{\mathcal{I}}$, for each $R \in \mathrm{N}_{\mathrm{R}}$,

- $\left(R^{-1}\right)^{\mathcal{I}}=\left(R^{\mathcal{I}}\right)^{-1}$, for each $R \in \mathrm{N}_{\mathrm{R}}$,

- a min-space $s^{\mathcal{I}}=\left(A_{s}^{\mathcal{I}}, d_{s}^{\mathcal{I}}\right)$ such that $\mathcal{D}_{1}(s)=A_{s} \in \mathrm{N}_{\mathrm{C}}, A_{s}^{\mathcal{I}} \neq \emptyset$, and $s^{\mathcal{I}}$ satisfies the constraints in $\mathcal{D}_{2}(s)$, for each $s \in \mathrm{S}$.

Given a $\Sigma$-type model $\mathcal{I}$, we define inductively the extension $C^{\mathcal{I}}$ of a concept $C$ :

$$
\begin{aligned}
&\{\ell\}^{\mathcal{I}}=\left\{\ell^{\mathcal{I}}\right\},(\neg C)^{\mathcal{I}}=\Delta^{\mathcal{I}} \backslash C^{\mathcal{I}}, \quad\left(C_{1} \sqcap C_{2}\right)^{\mathcal{I}}=C_{1}^{\mathcal{I}} \cap C_{2}^{\mathcal{I}}, \\
&(\leq n R . C)^{\mathcal{I}}=\left\{x \in \Delta^{\mathcal{I}}||\left\{y \in C^{\mathcal{I}} \mid x R^{\mathcal{I}} y\right\} \mid \leq n\right\}, \\
&(\geq n R . C)^{\mathcal{I}}=\left\{x \in \Delta^{\mathcal{I}}||\left\{y \in C^{\mathcal{I}} \mid x R^{\mathcal{I}} y\right\} \mid \geq n\right\}, \\
&\left(C_{1} \leftleftarrows{ }_{s} C_{2}\right)^{\mathcal{I}}=\left\{x \in A_{s}^{\mathcal{I}} \mid d_{s}^{\mathcal{I}}\left(x, C_{1}^{\mathcal{I}} \cap A_{s}^{\mathcal{I}}\right)<d_{s}^{\mathcal{I}}\left(x, C_{2}^{\mathcal{I}} \cap A_{s}^{\mathcal{I}}\right)\right\}, \\
&\left(\exists_{s}^{<a} C\right)^{\mathcal{I}}=\left\{x \in A_{s}^{\mathcal{I}} \mid \exists y \in\left(A_{s}^{\mathcal{I}} \cap C^{\mathcal{I}}\right) d_{s}^{\mathcal{I}}(x, y)<a\right\}, \\
&\left(\exists_{s}^{\leq a} C\right)^{\mathcal{I}}=\left\{x \in A_{s}^{\mathcal{I}} \mid \exists y \in\left(A_{s}^{\mathcal{I}} \cap C^{\mathcal{I}}\right) d_{s}^{\mathcal{I}}(x, y) \leq a\right\} .
\end{aligned}
$$

And if $T$ is a set of concepts, we write $T^{\mathcal{I}}$ for $\bigcap_{C \in T} C^{\mathcal{I}}$. Finally, a $\Sigma$-type model $\mathcal{I}$ satisfies a concept inclusion $C \sqsubseteq D$ iff $C^{\mathcal{I}} \subseteq D^{\mathcal{I}}$. It satisfies $\ell: C$ iff $\ell^{\mathcal{I}} \in C^{\mathcal{I}}, \ell_{1} R \ell_{2}$ iff $\ell_{1}^{\mathcal{I}} R^{\mathcal{I}} \ell_{2}^{\mathcal{I}}$, $d_{s}\left(\ell_{1}, \ell_{2}\right)<a$ iff $d_{s}^{\mathcal{I}}\left(\ell_{1}^{\mathcal{I}}, \ell_{2}^{\mathcal{I}}\right)<a$, and $d_{s}\left(\ell_{1}, \ell_{2}\right)=a$ iff $d_{s}^{\mathcal{I}}\left(\ell_{1}^{\mathcal{I}}, \ell_{2}^{\mathcal{I}}\right)=a$. A $\Sigma$-type model $\mathcal{I}$ satisfies $\Sigma$ if is satisfies all concept inclusions and assertions in $\Sigma$.

Example. A small part of a knowledge base representing the 'World of Whisky' in the language $\mathcal{S L}+\mathcal{A L C} \mathcal{Q I O}$ is given in the introduction. Here we show how this language can be used for prototype clustering. Suppose that we are given a similarity space defined on (the

\footnotetext{
${ }^{2}$ The logic of comparative similarity interpreted over arbitrary metric spaces has been considered in [31].
} 
extension of) a concept $D$, and we want to define new concepts $C_{i}$ representing those elements of the space that have some property $C$ and are more similar to the prototypical object $\ell_{i}$ (interpreted by $a_{i}$ ) than to the objects $\ell_{j}$ and $\ell_{k}$ (interpreted by $a_{j}$ and $a_{k}$, respectively), where $\{i, j, k\}=\{1,2,3\}$. The terms

$$
\left\{\ell_{i}\right\} \leftleftarrows\left\{\ell_{j}\right\} \sqcup\left\{\ell_{k}\right\}, \quad \text { for }\{i, j, k\}=\{1,2,3\},
$$

define the Voronoi tessellation of the space $D$ corresponding to the set $\left\{a_{1}, a_{2}, a_{3}\right\}$; see Fig. 1 where $D$ is interpreted by the Euclidean plane $\mathbb{R}^{2}$. The 'clusters' $C_{i}$ we need can be defined then as $C_{i}=C \sqcap\left(\left\{\ell_{i}\right\} \leftleftarrows\left\{\ell_{j}\right\} \sqcup\left\{\ell_{k}\right\}\right)$.

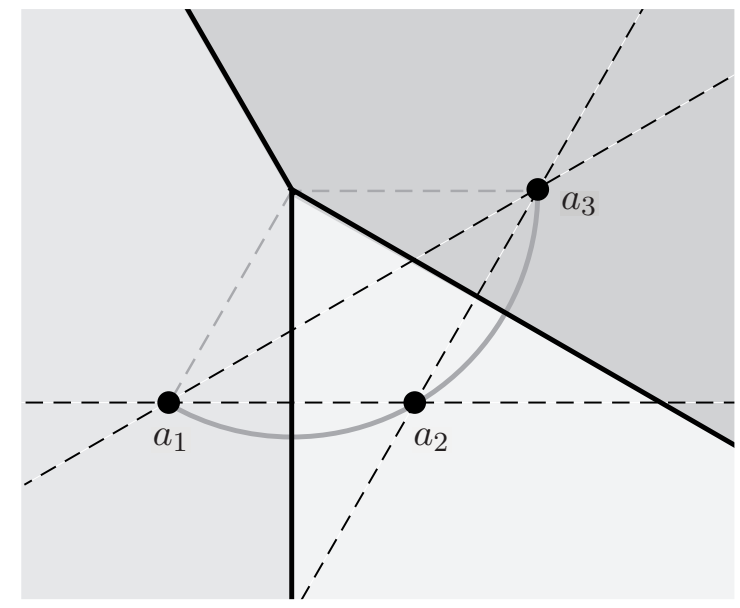

Figure 1: Closer operator and Voronoi tessellation.

Reasoning problems. The first important reasoning problem when dealing with a given $\mathrm{KB} \Sigma$ is its consistency, that is, the question:

- Is there a $\Sigma$-type model satisfying $\Sigma$ ?

Other standard reasoning tasks include:

- Concept satisfiability relative to a KB: given a concept $C$ and a $\mathrm{KB} \Sigma$, does there exist a $\Sigma$-type model $\mathcal{I}$ satisfying $\Sigma$ and such that $C^{\mathcal{I}} \neq \emptyset$ ?

- Subsumption relative to a KB: given a concept inclusion $C \sqsubseteq D$ and a $\mathrm{KB} \Sigma$, is it the case that every model $\mathcal{I}$ satisfying $\Sigma$ also satisfies $C \sqsubseteq D$ ?

- Instance checking relative to a KB: given a concept $C$, an object name $\ell$, and a $\mathrm{KB} \Sigma$, is it the case that every model $\mathcal{I}$ satisfying $\Sigma$ satisfies $\ell: C$ ?

All these tasks are reducible to each other. For example, there exists a model $\mathcal{I}$ satisfying $\Sigma$ where $C^{\mathcal{I}} \neq \emptyset$ iff $\Sigma \cup\{\ell: C\}$ is consistent, for some fresh object name $\ell$. Or, every model satisfying $\Sigma$ satisfies $\ell: C$ as well iff $\Sigma \cup\{\ell: \neg C\}$ is not consistent. 
Component logics. The logic $\mathcal{S L}+\mathcal{A L C} \mathcal{L I O}$ consists of two components. The first line of (1) defines $\mathcal{A L C} \mathcal{L I O}$ concepts. In every $\mathcal{A L C} \mathcal{L I O}$ knowledge base $\Sigma=\left(\mathcal{T}^{\Sigma}, \mathcal{A}^{\Sigma}, \mathcal{D}^{\Sigma}\right)$ the third component is empty and the first two are restricted to the language of $\mathcal{A L C Q \mathcal { O }}$.

The other component logic is the new similarity logic $\mathcal{S} \mathcal{L}$. Its concepts are constructed using nominals $\{\ell\}$, the Booleans $\sqcap, \neg$, and the similarity operators $\leftleftarrows, \exists<a$, and $\exists \leq a$. In fact, $\mathcal{S L}+\mathcal{A L C} \mathcal{Q I O}$ contains as many 'copies' of this logic as the cardinality of $\mathrm{S}$ (the number of similarity spaces) prescribes (we then use the subscript $s$ to distinguish between them).

An $\mathcal{S} \mathcal{L}$-model $\mathcal{I}$ is a distance space $\left(\Delta^{\mathcal{I}}, d^{\mathcal{I}}\right)$ together with interpretations $\ell^{\mathcal{I}}$ and $A^{\mathcal{I}}$ of the object and concept names. The extension $C^{\mathcal{I}}$ of an $\mathcal{S} \mathcal{L}$-concept $C$ is computed as defined above. An $\mathcal{S} \mathcal{L}$ knowledge base $\Sigma=\left(\mathcal{T}^{\Sigma}, \mathcal{A}^{\Sigma}, \mathcal{D}^{\Sigma}\right)$ is restricted to the language of $\mathcal{S} \mathcal{L}$ and $\mathcal{D}^{\Sigma}$ contains just a single similarity space.

\section{$3 \quad$ Decidability and complexity of $\mathcal{S} \mathcal{L}+\mathcal{A L C} \mathcal{L I O}$}

The first main result of this paper is the following

Theorem 1. The consistency and concept satisfiability problems formulated above for $\mathcal{S} \mathcal{L}+$ $\mathcal{A L C} \mathcal{Q I O}$ KBs $\Sigma$ are NExpTime-complete. The subsumption and instance checking problems (relative to knowledge bases) are coNExp Time-complete. These complexity results hold for both unary and binary codings of the numerical parameters in number restrictions and similarity operators.

The NExpTime lower bound under unary coding of parameters follows from the fact that already consistency of $\mathcal{A L C} \mathcal{Q I O}$ knowledge bases is NExpTime-hard [34]. In what follows we, therefore, concentrate on the corresponding upper bound under the binary coding of parameters.

An almost obvious idea of how to prove this theorem would be to try to decompose $\mathcal{S L}+\mathcal{A L C Q \mathcal { O } O}$ into the fusion [4] of its component logics. Indeed, one can polynomially reduce the reasoning problems for $\mathcal{S}+\mathcal{A L C} \mathcal{Q I O}$ to the corresponding problems for the fusion of the description logic $\mathcal{A L C} \mathcal{Q} \mathcal{I O}$ and the similarity logics $\mathcal{S} \mathcal{L}_{s}, s \in \mathrm{S}$. But unfortunately such a reduction does not help to determine the computational complexity of the reasoning tasks. What is more important is that the reduction does not even provide us with a decidability proof. The reason is that all known transfer results for fusions, with the exception of [11], rely upon the assumption that the component languages do not contain nominals. In the language we consider here, however, the presence of nominals is crucial (to model, for example, prototypical reasoning). The transfer result of [11] does apply to logics with nominals. Yet, we cannot use it for our logic because it makes the strong assumption that there are no 'hidden' nominals in the components in the sense that they must not be able to express, using a TBox, that certain concepts $C$ have to be interpreted as singleton sets unless there actually exists an object name $\ell$ such that $\{\ell\} \equiv C$ follows from the TBox (this property is called nominal-closedness in [11]). The logic $\mathcal{A L C} \mathcal{L I O}$ does not have this property. Indeed, consider the TBox $\mathcal{T}$ with two axioms

$$
A \equiv\left(=1 R^{-1} \cdot\{\ell\}\right), \quad\{\ell\} \equiv(=1 R \cdot A),
$$

where $(=1 R . C)$ stands for $(\leq 1 R . C) \sqcap(\geq 1 . R . C)$. Then, in every model of $\mathcal{T}, A$ is interpreted as a singleton set, but there is no nominal $\left\{\ell^{\prime}\right\}$ such that $\left\{\ell^{\prime}\right\} \equiv A$ follows from $\mathcal{T}$. 
In spite of these difficulties, we show in the remaining part of this section that a NExpTime decision procedure for $\mathcal{S L}+\mathcal{A L C Q \mathcal { O }}$ can be obtained as a modular combination of an existing decision procedure for $\mathcal{A L C Q \mathcal { O } O}$ (actually, we take a decision procedure for $C^{2}$, the two-variable fragment of first-order logic with counting [26]) and a decision procedure for $\mathcal{S} \mathcal{L}$ developed in Section 4.

Before turning on to technical details, we observe that without loss of generality we can always assume that the ABox of a given $\mathrm{KB}$ is empty. Indeed, the assertion $\ell: C$ is equivalent to $\{\ell\} \sqsubseteq C, \ell_{1} R \ell_{2}$ is equivalent to $\left\{\ell_{1}\right\} \sqsubseteq \exists R .\left\{\ell_{2}\right\}, d_{s}\left(\ell_{1}, \ell_{2}\right)<a$ is equivalent to $\left\{\ell_{1}\right\} \sqsubseteq \exists_{s}^{<a}\left\{\ell_{2}\right\}$, and $d_{s}\left(\ell_{1}, \ell_{2}\right)=a$ is equivalent to $\left\{\ell_{1}\right\} \sqsubseteq \exists s a\left\{\ell_{2}\right\},\left\{\ell_{1}\right\} \sqsubseteq \neg \exists_{s}^{<a}\left\{\ell_{2}\right\}$. In what follows we always assume that our knowledge bases have empty ABoxes.

The decision procedure for $\mathcal{S} \mathcal{L}+\mathcal{A L C} \mathcal{Q} \mathcal{I O}$. We now show how the consistency problem for an arbitrary KB $\Sigma$ with empty ABox can be split into the consistency problems for $\mathcal{S} \mathcal{L}$ and $\mathcal{A L C} \mathcal{Q I O}$ knowledge bases.

Let $S$ be the set of names for similarity spaces in $\Sigma$. Define the length $l(\Sigma)$ of $\Sigma$ by taking

$$
l(\Sigma)=|\mathrm{S}|+\sum_{C \sqsubseteq D \in \mathcal{T}^{\Sigma}}(l(C)+l(D))
$$

where $l(C)$ is the length of $C$ under the binary coding of numerical parameters. Let $\mathrm{P}(\Sigma)$ be the set of all rational numbers (we will call them parameters) occurring in the similarity operators $\left(\exists_{s}^{<a}\right.$ and $\left.\exists_{s}^{\leq a}\right)$ from $\Sigma$, and let

$$
\mathrm{P}^{+}(\Sigma)=\left\{b=a_{1}+\cdots+a_{n} \mid a_{i} \in \mathrm{P}(\Sigma), b \leq \max \mathrm{P}(\Sigma)\right\} .
$$

We have $|P(\Sigma)|<l(\Sigma)$ and $\left|\mathrm{P}^{+}(\Sigma)\right| \leq \max P(\Sigma) / \operatorname{gcd} P(\Sigma)$ (where $\operatorname{gcd} P(\Sigma)$ is the greatest common divisor of the numbers in $P(\Sigma)$ ). Therefore $\left|\mathrm{P}^{+}(\Sigma)\right|<2^{l(\Sigma)}$. Set $\exists_{s} D=D \leftleftarrows_{s} \perp$. Note that $\exists_{s}$ behaves like the existential modality on the spaces $A_{s}^{\mathcal{I}}[12]$, that is, $\left(\exists_{s} D\right)^{\mathcal{I}}=A_{s}^{\mathcal{I}}$ if $D^{\mathcal{I}} \cap A_{s}^{\mathcal{I}} \neq \emptyset$, and $\left(\exists_{s} D\right)^{\mathcal{I}}=\emptyset$ otherwise.

Denote by $\operatorname{cl}(\Sigma)$ the smallest set of concepts containing all concepts from $\Sigma$ and closed under the following rules:

- $\operatorname{cl}(\Sigma)$ is closed under taking subconcepts and single negation,

- if $D_{1} \leftleftarrows{ }_{s} D_{2} \in \operatorname{cl}(\Sigma)$ then $\exists_{s} D_{1}, \exists_{s} D_{2} \in \operatorname{cl}(\Sigma)$,

- if $\exists_{s}^{<a} D \in \operatorname{cl}(\Sigma)$ or $\exists \frac{\leq a}{s} D \in \operatorname{cl}(\Sigma)$ then $\exists_{s} D \in \operatorname{cl}(\Sigma)$,

- if $\exists_{s} D \in \operatorname{cl}(\Sigma)$ and $a \in \mathrm{P}^{+}(\Sigma)$ then $\exists_{s}^{<a} D, \exists_{s}^{\leq a} D \in \operatorname{cl}(\Sigma)$.

Then the size of $\operatorname{cl}(\Sigma)$ is at most exponential in $l(\Sigma)$. Indeed, $\operatorname{cl}(\Sigma)$ can be constructed in the following way: we take $\operatorname{sub}(\Sigma)$, the closure $\Sigma$ under subconcepts, then add the required terms of the form $\exists_{s} D, \exists_{s}^{\leq a} D$, and $\exists_{s}^{<a} D$, and finally close the resulting set under single negations. Thus, we have

$$
|\operatorname{cl}(\Sigma)| \leq l(\Sigma) \cdot\left(2\left|\mathrm{P}^{+}(\Sigma)\right|+1\right) \cdot 2 \leq 4 \cdot l(\Sigma) \cdot 2^{l(\Sigma)} .
$$

A very important notion in our construction is that of a type over $\Sigma$. Namely, a type over $\Sigma$ is a subset $T$ of $\operatorname{cl}(\Sigma)$ such that the following conditions are satisfied:

- $D_{1} \sqcap D_{2} \in T$ iff $D_{1}, D_{2} \in T$, for every $D_{1} \sqcap D_{2} \in \operatorname{cl}(\Sigma)$,

- $\neg D \in T$ iff $D \notin T$, for every $\neg D \in \operatorname{cl}(\Sigma)$, 
- if $\exists_{s}^{<a} D \in T$ then $\exists \leq a, D \in T$,

- if $\exists_{s}^{\leq a} D \in T$ then $\exists_{s}^{<b} D, \exists_{s}^{\leq b} D, \exists_{s} D \in T$ for all $b>a$ in $\mathrm{P}^{+}(\Sigma)$,

- if $D_{1} \leftleftarrows_{s} D_{2} \in T$ and $\exists_{s}^{\leq a} D_{2} \in T$ then $\exists_{s}^{<a} D_{1} \in T$,

- if $\exists_{s} D \in T$ for some $D$, then $A_{s} \in T$.

It is to be noted that although the size of $\operatorname{cl}(\Sigma)$ can be exponential in $l(\Sigma)$, we still have at most exponentially many distinct types over $\Sigma$. This follows from the fact that, for any type $T$ and any $\exists_{s} D \in T$, there is always exactly one $\zeta$ in the list

$$
\leq 0,<a, \leq a\left(\text { for } a \in \mathrm{P}^{+}(\Sigma)\right),<\infty
$$

such that $\exists_{s}^{\zeta} D$ belongs to $T$ and we have $\exists_{s}^{\xi} D \in T$ for every $\xi$ that is 'weaker' than this $\zeta$ (we naturally assume here that $\exists_{s}^{\leq 0} D=D, \exists_{s}^{<\infty} D=\exists_{s} D$, and $\leq a$ is stronger than $<b$, which is stronger than $\leq b$, for all $a<b$ ). Therefore, every type is determined by the following information, for every $D \in \operatorname{sub}(\Sigma)$ : (i) whether $D$ belongs to $T$, and (ii) the strongest $\zeta$ in (2) with $\exists_{s}^{\zeta} D \in T$, whenever $\exists_{s} D \in T$. Hence, the number of types over $\Sigma$ is bounded by

$$
\left(2\left|\mathrm{P}^{+}(\Sigma)\right|+2\right)^{|\operatorname{sub}(\Sigma)|} \leq\left(2 \cdot 2^{l(\Sigma)}\right)^{l(\Sigma)} .
$$

Given an interpretation $\mathcal{I}$ and an element $u$ of its domain, define the type $T^{\mathcal{I}}(u)$ of $u$ in $\mathcal{I}$ by taking

$$
T^{\mathcal{I}}(u)=\left\{C \in \operatorname{cl}(\Sigma) \mid u \in C^{\mathcal{I}}\right\} .
$$

It is easily seen that this is indeed a type over $\Sigma$. We remind the reader that types of a similar form are basic ingredients of various worst-case optimal algorithms in description, modal, and temporal logics $[36,25]$. In the definition above, however, we had to be particularly careful in order to avoid creating double-exponentially many types.

To design a modular consistency checking algorithm for $\mathcal{S L}+\mathcal{A L C} \mathcal{Q} \mathcal{I O}$ knowledge bases, we define 'abstractions' of concepts that will be used as inputs for the component algorithms. For each $C$ of the form $\exists_{s}^{<a} D, \exists_{s}^{\leq a} D, D_{1} \leftleftarrows_{s} D_{2}, \leq n R . D$ and $\geq n R . D$ we reserve a fresh concept name $A^{C}$, the surrogate of $C$. Now, given an arbitrary concept $C$, denote by $C^{\mathcal{D} L}$ the result of replacing in $C$ all outermost occurrences of subconcepts of the form $\exists_{s}^{<a} D$, $\exists_{s}^{\leq a} D, D_{1} \leftleftarrows_{s} D_{2}$ with their surrogates. Let $\operatorname{cl}(\Sigma)^{\mathcal{D L}}$ (or $T^{\mathcal{D L}}$ ) be the set of all $C^{\mathcal{D} \mathcal{L}}$ such that $C \in \operatorname{cl}(\Sigma)$ (respectively, $C \in T$ ).

Similarly, for each $s \in \mathrm{S}$ and each concept $C$, denote by $C^{s}$ the result of replacing in $C$ all outermost occurrences of subconcepts of the form $\leq n R . D, \geq n R . D, \exists_{s^{\prime}}^{<a} D, \exists_{s^{\prime}}^{\leq a} D, D_{1} \leftleftarrows_{s^{\prime}} D_{2}$, for $s^{\prime} \neq s$, with their surrogates. Note that $\operatorname{cl}(\Sigma)^{\mathcal{D \mathcal { L }}}=\operatorname{cl}\left(\Sigma^{\overline{\mathcal{D}}}\right), \operatorname{cl}(\Sigma)^{s}=\operatorname{cl}\left(\Sigma^{s}\right)$, and if $T$ is a type over $\operatorname{cl}(\Sigma)$, then $T^{\mathcal{D L}}$ and $T^{s}$ are types over $\operatorname{cl}(\Sigma)^{\mathcal{D} \mathcal{L}}$ and $\operatorname{cl}(\Sigma)^{s}$, respectively.

Say that a set $\mathcal{T}$ of types over $\operatorname{cl}(\Sigma)^{\mathcal{D L}}$ is $\mathcal{A L C} \mathcal{Q} \mathcal{I O}$-realisable if there exists an interpretation $\mathcal{I}$ such that

$$
\mathcal{T}=\left\{T^{\mathcal{I}}(u) \mid u \in \Delta^{\mathcal{I}}\right\}
$$

Similarly, we say that a set $\mathcal{T}$ of types over $\operatorname{cl}(\Sigma)^{s}$ is $\mathcal{S} \mathcal{L}$-realisable if there exists an $\mathcal{S} \mathcal{L}$-model $\mathcal{I}$ with domain $A_{s}^{\mathcal{I}}$ such that (3) holds.

A modular nondeterministic consistency checking procedure for knowledge bases over $\mathcal{S L}+\mathcal{A L C} \mathcal{Q I O}$ is sketched in Fig. 2.

It is easily seen that if there is no set $\mathcal{T}$ with the properties listed in Fig. 2, then $\Sigma$ is inconsistent. Indeed, suppose that $\Sigma$ is consistent. Take a $\Sigma$-type model $\mathcal{I}$ satisfying $\Sigma$. 
Given a knowledge base $\Sigma$ (without an ABox),

1. Guess a nonempty set $\mathcal{T}$ of types over $\Sigma$.

2. Check whether the following conditions hold:

- if $C \sqsubseteq D \in \mathcal{T}^{\Sigma}$ then $\neg C \in T$ or $D \in T$, for all $T \in \mathcal{T}$,

- if $\exists_{s} D \in T$ for some $T \in \mathcal{T}$, then $\exists_{s} D \in T^{\prime}$ for all $T^{\prime} \in \mathcal{T}$ such that $A_{s} \in T^{\prime}$.

3. Check whether the set $\left\{T^{\mathcal{D L}} \mid T \in \mathcal{T}\right\}$ of types over $\operatorname{cl}(\Sigma)^{\mathcal{D L}}$ is $\mathcal{A L C} \mathcal{Q} \mathcal{I} \mathcal{O}$-realisable.

4. For each $s \in \mathrm{S}$, check whether the set $\left\{T^{s} \mid T \in \mathcal{T}, A_{s} \in T\right\}$ of types over $\mathrm{cl}(\Sigma)^{s}$ is $\mathcal{S} \mathcal{L}$-realisable in an interpretation satisfying the constraints in $\mathcal{D}_{2}(s)$.

5. If the answer is YES for at least one guessed set $\mathcal{T}$ of types over $\Sigma$, then $\Sigma$ is consistent; otherwise it is inconsistent.

Figure 2: Decision procedure.

Define $\mathcal{T}$ by (3). It is straightforward to see that for this set $\mathcal{T}$ the algorithm in Fig. 2 returns the answer YES.

The converse direction as well as the proof that this algorithm runs in NExpTime are based on two theorems. The first one shows that item 3 in Fig. 2 can be checked in NExpTime:

Theorem 2. It is decidable in NExpTime (in the size of $\Sigma$ ) whether a set $\mathcal{T}$ of types over an $\mathcal{A L C} \mathcal{L I O}$ knowledge base $\Sigma$ is realisable. This holds for the binary coding of the numerical parameters in the qualified number restriction operators.

Proof. It is well known that $\mathcal{A L C} \mathcal{Q I O}$ can be regarded as a fragment of $C^{2}$, the two-variable fragment of first-order logic with counting [2, page 152]. A recent result of Pratt-Hartmann [26] shows that the satisfiability problem for $C^{2}$-formulas is NExpTime-complete under the binary coding of counting quantifiers. We cannot apply this result directly to the problem stated in the theorem because the $C^{2}$-formula encoding the realisability of $\mathcal{T}$ is already exponential in the size $l(\Sigma)$ of $\Sigma$. However, Pratt-Hartmann's proof is type-based in the sense that in order to decide (in NExpTime) whether a $C^{2}$-formula $\varphi$ is satisfiable, it guesses a set of types over the formula $\varphi$ (together with some additional information about the types) and then checks whether this set of types is realisable (again together with a number of additional constraints). A close inspection of Pratt-Hartmann's procedure shows that it still runs in NExpTime if the input is not a formula but a set of types whose realisability is to be checked. We now sketch this reduction to Pratt-Hartmann's proof in more detail. For $C^{2}$ we use the notation of [26], which the reader should have at hand.

Suppose that $\mathcal{T}$ is a set of types over an $\mathcal{A L C} \mathcal{Q I O}$ knowledge base $\Sigma_{0}$ (here we use $\Sigma_{0}$ rather than $\Sigma$ because Pratt-Hartmann uses the symbol $\Sigma$ to denote a certain signature). Instead of 1-types over arbitrary $C^{2}$-formulas with one free variable $x$, Pratt-Hartmann uses 1-types over literals with the free variable $x$. Therefore, we first reformulate the problem of checking realisability of a set of types as the problem of realising a set of types over literals together with a formula. For every non-atomic concept $C$, which does not start with a negation symbol, we introduce a new atomic concept $A_{C}$, and set $A_{C}=C$ for every atomic $C$ in $\operatorname{cl}\left(\Sigma_{0}\right)$. Now define, for every concept $C$ in $\operatorname{cl}\left(\Sigma_{0}\right)$, a concept $C^{s}$ by taking $C^{s}=A_{C}$ if $C$ does not start with a negation symbol, and $C^{s}=\neg A_{D}$ if $C=\neg D$. For $T \in \mathcal{T}$, we then set 
$T^{s}=\left\{C^{s} \mid C \in T\right\}$. Let $\Gamma$ be the collection of the following TBox axioms:

$$
A_{\{\ell\}} \equiv\{\ell\}, \quad A_{D_{1} \sqcap D_{2}} \equiv D_{1}^{s} \sqcap D_{2}^{s}, \quad A_{\leq n R . D} \equiv\left(\leq n R \cdot D^{s}\right), \quad A_{\geq n R . D} \equiv\left(\geq n R \cdot D^{s}\right),
$$

where $\{\ell\}, D_{1} \sqcap D_{2}, \leq n R . D, \geq n R . D \in \operatorname{cl}(\Sigma)$. It can be shown that there exists a model realising $\mathcal{T}$ iff there exists a model realising $\left\{T^{s} \mid T \in \mathcal{T}\right\}$ and satisfying $\Gamma$. Observe that the types in $\left\{T^{s} \mid T \in \mathcal{T}\right\}$ contain literals only. We remind the reader of the standard translation from $\mathcal{A L C Q \mathcal { I O }}$ concepts into $C^{2}$ (with two variables $x, y$ ). First, we may regard atomic concepts $A$ of $\mathcal{A L C Q \mathcal { O }}$ as unary predicate symbols and roles names $R$ as binary predicate symbols. Now we define inductively a translation $C^{\sharp}$ of an $\mathcal{A L C} \mathcal{Q I O}$-concept $C$ by taking:

$$
\begin{aligned}
& \{\ell\}^{\sharp}=A_{\{\ell\}}(x), \quad A^{\sharp}=A(x), \quad(\neg C)^{\sharp}=\neg C^{\sharp}, \quad\left(C_{1} \sqcap C_{2}\right)^{\sharp}=C_{1}^{\sharp} \wedge C_{2}^{\sharp}, \\
& (\leq n R . C)^{\sharp}=\exists^{\leq n} y\left(R(x, y) \wedge C^{\sharp}[y / x]\right), \quad(\geq n R . C)^{\sharp}=\exists^{\geq n} y\left(R(x, y) \wedge C^{\sharp}[y / x]\right) .
\end{aligned}
$$

Then there exists a model realising $\left\{T^{s} \mid T \in \mathcal{T}\right\}$ and satisfying $\Gamma$ iff there exists a model (for $C^{2}$ ) which satisfies exactly the 1-types

$$
\tau=\left\{\left\{C^{\sharp}(x) \mid C \in T^{s}\right\} \mid T \in \mathcal{T}\right\}
$$

and also satisfies

$$
\Phi=\left\{\forall x\left(C^{\sharp}(x) \leftrightarrow D^{\sharp}(x)\right) \mid C \equiv D \in \Gamma\right\} \cup\left\{\exists^{=1} A_{\{\ell\}}(x) \mid\{\ell\} \text { occurs in } \operatorname{cl}\left(\Sigma_{0}\right)\right\} .
$$

Thus, it remains to show that we can decide the latter problem in NExpTime. Suppose that $\phi=\bigwedge \Phi$ and $\tau$ are given. Observe that $\bigwedge \Phi$ is polynomial in $l\left(\Sigma_{0}\right)$. Compute $\phi^{*}$ as in $[26$, Lemma 1] and take the number $C$ from that lemma, which will be denoted here (for obvious reasons) by $M$. An inspection of the proof of [26, Lemma 1] shows that there exists a model with domain of size $\geq M$ satisfying exactly the 1-types in $\tau$ and $\phi$ iff there exists a model of size $\geq M$ satisfying exactly the types in $\tau$ and $\phi^{*}$.

Clearly, satisfiability of exactly the types in $\tau$ and $\phi$ in a model of size $\leq M$ is decidable in NExpTime. Thus, it remains to show that satisfiability of exactly the types in $\tau$ and $\phi^{*}$ in a model is decidable in NExpTime. (Observe that $\phi^{*}$ is a $C^{2}$-formula over a larger signature than $\phi$. Thus, the 1-types over this signature are sets properly containing 1-types over the signature of $\phi$ and, in particular, the 1-types from $\tau$.)

Now, in the proof of [26, Theorem 2] it is shown that $\phi^{*}$ is satisfiable iff $(\dagger)$ there exists a chromatic, $M$-bounded frame $\mathcal{F}$ over a certain signature $\Sigma$ extending the signature of $\phi^{*}$ of dimension $N \leq X$ (for the number $X$ defined in [26]), such that $\mathcal{F}$ has an extended solution and $\mathcal{F} \models \phi^{*}$. Condition $(\dagger)$ can be checked in NExpTime. The important observation for us is that a frame $\mathcal{F}=(\bar{\sigma}, I, \theta)$ consists of a sequence $\bar{\sigma}=\left(\sigma_{1}, \ldots, \sigma_{N}\right)$ of so-called star-types, where a star-type $\sigma$ is a pair $(\pi, \bar{v})$ in which $\pi$ is a 1-type, and that Pratt-Hartmann actually proves that there is a witness frame $\mathcal{F}$ for $(\dagger)$ iff there exists a model satisfying $\phi^{*}$ which satisfies exactly the 1-types occurring in the sequence of star-types of $\mathcal{F}$. It follows that there exists a model satisfying exactly the types in $\tau$ and $\phi^{*}$ iff there exists a chromatic, $M$-bounded frame $\mathcal{F}$ over a certain signature $\Sigma$ extending the signature of $\phi^{*}$ of dimension $N \leq X$, such that $\mathcal{F}$ has an extended solution, $\mathcal{F} \models \phi^{*}$, and

- every 1-type from a star-type in $\mathcal{F}$ contains a 1-type from $\tau$;

- for every 1-type from $\tau$, there exists a 1-type from a star-type in $\mathcal{F}$ which contains $\tau$. 
These two additional conditions can be checked in exponential time (in $\Sigma_{0}$ ), and it follows that checking $(\dagger)$ with those two additional conditions on the frame $\mathcal{F}$ is decidable in NExpTime as well.

We now formulate the second main result of this paper which gives an ExpTime decision procedure for the similarity logic $\mathcal{S} \mathcal{L}$. It also establishes in (ii) that item 4 in Fig. 2 can be checked in ExpTime. Under (iii) it lists the property required to show the correctness of our algorithm. In fact, (iii) is the crucial property of $\mathcal{S} \mathcal{L}$ which enables us to provide a modular decision procedure despite the fact that $\mathcal{A L C} \mathcal{L I O}$ is not nominal-closed. Roughly speaking, it states that satisfiable types, which do not contain a nominal, can be satisfied in an arbitrary number of points in a model.

Theorem 3. (i) The consistency problem for $\mathcal{S} \mathcal{L}$ knowledge bases is ExpTime-complete for the binary coding of the numerical parameters in the similarity operators.

(ii) It is decidable in ExpTime (in the size of $\Sigma$ ) whether a set $\mathcal{T}$ of types over an $\mathcal{S} \mathcal{L}$ knowledge base $\Sigma$ is realisable in a model satisfying the given constraints from $\{\mathrm{sym}, \mathrm{tr}\}$. This holds for the binary coding of the numerical parameters in the similarity operators.

(iii) Moreover, let $T_{1}, \ldots, T_{n}$ be an enumeration of all those types from $\mathcal{T}$ that do not contain nominals, and let $k_{1}, \ldots, k_{n}$ be a sequence of some positive numbers from $\mathbb{N} \cup\{\omega\}$. If $\mathcal{T}$ is realisable then it is realisable in a model $\mathcal{I}$ such that $\left|T_{1}^{\mathcal{I}}\right|=k_{1}, \ldots,\left|T_{n}^{\mathcal{I}}\right|=k_{n}$.

A proof of this theorem will be given in Section 4 .

Let us see now that the algorithm in Fig. 2 is correct. Suppose that the procedure returns 'YES' for some set $\mathcal{T}$ of types over $\Sigma$.

By item 3 in Fig. 2, we find an interpretation $\mathcal{I}$ which realises $\left\{T^{\mathcal{D} \mathcal{L}} \mid T \in \mathcal{T}\right\}$. As $\mathcal{A L C} \mathcal{L I O}$ is a fragment of first-order logic, we can assume that $\Delta^{\mathcal{I}}$ is countable.

Consider any $s \in \mathrm{S}$. Let $\mathcal{T}_{s}=\left\{T \in \mathcal{T} \mid A_{s} \in T\right\}$ and $\mathcal{T}^{s}=\left\{T^{s} \mid T \in \mathcal{T}, A_{s} \in T\right\}$. By item 4 in Fig. 2, $\mathcal{T}^{s}$ is realisable. And we see that, for each $T \in \mathcal{T}$, either the types $T^{s}$ and $T^{\mathcal{D} \mathcal{L}}$ both contain a nominal $\{\ell\}$ or neither of them contains $\{\ell\}$. Therefore, by Theorem 3 (iii), there exists a model $\mathcal{I}_{s}$ realising $\mathcal{T}^{s}$ such that $\left|\left(T^{s}\right)^{\mathcal{I}_{s}}\right|=\left|\left(T^{\mathcal{D} \mathcal{L}}\right)^{\mathcal{I}}\right|$, for all $T \in \mathcal{T}_{s}$.

For every pair $(s, T)$ with $T \in \mathcal{T}_{s}$, take a bijection $f_{s, T}$ from $\left(T^{s}\right)^{\mathcal{I}_{s}}$ onto $\left(T^{\mathcal{D} \mathcal{L}}\right)^{\mathcal{I}}$ and let

$$
f_{s}=\bigcup_{T \in \mathcal{T}_{s}} f_{s, T}
$$

Then $f_{s}$ is a bijection from $\Delta^{\mathcal{I}_{s}}$ onto $A_{s}^{\mathcal{I}}$ (recall that every point satisfies exactly one type). Consider any $C \in \operatorname{cl}(\Sigma)$ and $s \in \mathrm{S}$, and let $\mathcal{T}^{\prime}=\left\{T \in \mathcal{T} \mid C, A_{s} \in T\right\}$. Then $\left(C^{s}\right)^{\mathcal{I}_{s}}=$ $\bigcup_{T \in \mathcal{T}^{\prime}}\left(T^{s}\right)^{\mathcal{I}_{s}}$ and $\left(C^{\mathcal{D L}}\right)^{\mathcal{I}}=\bigcup_{T \in \mathcal{T}^{\prime}}\left(T^{\mathcal{D L}}\right)^{\mathcal{I}}$. Hence, for all $s \in \mathrm{S}$ and $C \in \operatorname{cl}(\Sigma)$,

$$
\left(C^{\mathcal{D L}}\right)^{\mathcal{I}} \cap A_{s}^{\mathcal{I}}=f_{s}\left(\left(C^{s}\right)^{\mathcal{I}_{s}}\right) .
$$

Suppose now that $C$ is of the form $\exists_{s}^{<a} D, \exists_{s}^{\leq a} D$, or $D \leftleftarrows_{s} D^{\prime}$. Then, by the definition of a type, every $T \in \mathcal{T}$ with $C \in T$ contains $A_{s}$. Moreover, $C \in T$ iff $A^{C} \in T^{\mathcal{D L}}$. Since $\mathcal{I}$ realises $\mathcal{T}^{\mathcal{D} \mathcal{L}}$, and $\mathcal{I}_{s}$ realises $\mathcal{T}^{s}$, we obtain $\left(A^{C}\right)^{\mathcal{I}} \subseteq\left(A_{s}\right)^{\mathcal{I}}$.

Now define a new model $\mathcal{J}$ with domain $\Delta^{\mathcal{I}}$ by taking the interpretation of atomic concepts and role names from $\mathcal{I}$ and defining, for all $s \in \mathrm{S}$ and $x, y \in A_{s}^{\mathcal{I}}$,

$$
d_{s}^{\mathcal{J}}(x, y)=d_{s}^{\mathcal{I}_{s}}\left(f_{s}^{-1}(x), f_{s}^{-1}(y)\right) .
$$


We prove by induction that $C^{\mathcal{J}}=\left(C^{\mathcal{D} \mathcal{L}}\right)^{\mathcal{I}}$, for all $C \in \operatorname{cl}(\Sigma)$. The basis of induction and the case of Booleans are obvious.

Let $C=\leq n R . D$. Then $R^{\mathcal{J}}=R^{\mathcal{I}}$ and $D^{\mathcal{J}}=D^{\mathcal{I}}$ by the construction of $\mathcal{J}$ and the induction hypothesis. Now $C^{\mathcal{J}}=\left(C^{\mathcal{D L}}\right)^{\mathcal{I}}$ follows immediately from the truth condition for $\leq n R$. The case $C=\geq n R$. $D$ is considered in the same way.

Let $C=\exists_{s}^{<a} D$. Then $C^{s}=\exists_{s}^{<a} D^{s}, C^{\mathcal{D} \mathcal{L}}=A^{C}$, and $\left(A^{C}\right)^{\mathcal{I}} \subseteq A_{s}^{\mathcal{I}}$. Hence, in view of (4), it suffices to prove that $C^{\mathcal{J}}=f_{s}\left(\left(C^{s}\right)^{\mathcal{I}_{s}}\right)$. By the induction hypothesis and (4), we have $D^{\mathcal{J}} \cap A_{s}^{\mathcal{J}}=\left(D^{\mathcal{D L}}\right)^{\mathcal{I}} \cap A_{s}^{\mathcal{I}}=f_{s}\left(\left(D^{s}\right)^{\mathcal{I}_{s}}\right)$. Therefore, by the truth condition for $\exists_{s}^{<a}$ and the definition of $d^{\mathcal{J}}$,

$$
\begin{aligned}
u \in C^{\mathcal{J}} \text { iff } \exists v \in\left(D^{\mathcal{J}} \cap A_{s}^{\mathcal{J}}\right) d_{s}^{\mathcal{J}}(u, v)<a \\
\text { iff } \exists v \in f_{s}\left(\left(D^{s}\right)^{\mathcal{I}_{s}}\right) d_{s}^{\mathcal{I}_{s}}\left(f^{-1}(u), f^{-1}(v)\right)<a \\
\text { iff } \exists w \in\left(D^{s}\right)^{\mathcal{I}_{s}} d_{s}^{\mathcal{I}_{s}}\left(f^{-1}(u), w\right)<a \text { iff } f^{-1}(u) \in\left(C^{s}\right)^{\mathcal{I}_{s}} .
\end{aligned}
$$

The cases $C=\exists_{s}^{\leq a} D$ and $C=D \leftleftarrows{ }_{s} D^{\prime}$ are considered similarly.

Thus, $\mathcal{J}$ realises $\mathcal{T}$. And for every subsumption $C \sqsubseteq D \in \mathcal{T}^{\Sigma}$, we have that $\neg C \in T$ or $D \in T$, by the first condition of item 2 in Fig. 2. This means that $\mathcal{J}$ satisfies $\Sigma$.

\section{The similarity logic $\mathcal{S} \mathcal{L}$}

In this section we prove Theorem 3. Recall that in $\mathcal{S L}$ we do not have qualified number restrictions. Moreover, we deal with only one similarity space, and so can omit indexes from the distance operators and write simply $\exists^{<a}, \exists \leq a, \leftleftarrows$.

An $\mathcal{S} \mathcal{L}$-model $\mathcal{I}$ was defined as a min-space $\left(\Delta^{\mathcal{I}}, d^{\mathcal{I}}\right)$ together with interpretations $\ell^{\mathcal{I}}$ and $A^{\mathcal{I}}$ of the object and concept names. We are going to prove Theorem 3 for two types of $\mathcal{S} \mathcal{L}$ knowledge bases $\Sigma$ :

- the non-symmetric case: $\mathcal{D}^{\Sigma}$ states that the min-spaces to be considered satisfy the triangle inequality only,

- the symmetric case: $\mathcal{D}^{\Sigma}$ states that the min-spaces to be considered are actually metric spaces (satisfying both (tr) and (sym)).

The proofs for the remaining two cases are easily obtained by a straightforward modification (in fact, simplification because the closer operator $\leftleftarrows$ does not 'feel' the triangle inequality; see [30]) of the proofs for the cases above (for example, we only need the first lines of (7) and (8), and only items (2) in Lemmas 5 and 9; condition 4.3 should also be removed from the definition of a bouquet).

For all possible types of constraints, the ExpTime lower bound required for Theorem 3 (i) was proved in [30] even for the language without nominals and operators of the form $\exists<a$ and $\exists \leq a$. The proof was by reduction of the global consequence relation for the modal logic $\mathbf{K}$ which is known to be ExpTime-hard [32]. Another encoding of the global consequence relation for $\mathbf{K}$ using the operator $\exists \leq 1$ and the Booleans can be found in [39].

In order to ease presentation, in the proof of the ExpTime upper bound we will consider the satisfiability problem for $\mathcal{S} \mathcal{L}$-concepts instead of the consistency problem for $\mathcal{S} \mathcal{L}$-knowledge bases. We call an $\mathcal{S} \mathcal{L}$-concept $C$ satisfiable in a class $\mathcal{M}$ of $\mathcal{S} \mathcal{L}$-models if there exists $\mathcal{I} \in \mathcal{M}$ such that $C^{\mathcal{I}} \neq \emptyset$. To see that consistency of $\mathcal{S} \mathcal{L}$-knowledge bases is reducible to satisfiability of $\mathcal{S} \mathcal{L}$-concepts, recall first that we may assume that the ABox of $\Sigma$ is empty. Also recall the 
definitions of the 'universal modalities:' $\exists D=D \leftleftarrows \perp$ and $\forall D=\neg \exists \neg D$. Now, $\Sigma$ is consistent iff $\forall C$ is satisfiable in a model from the class $\mathcal{M}$ of all $\mathcal{S} \mathcal{L}$ models meeting the constraints in $\mathcal{D}^{\Sigma}$, where $C=\prod\left\{\neg D_{1} \sqcup D_{2} \mid D_{1} \sqsubseteq D_{2} \in \mathcal{T}^{\Sigma}\right\}$. The statements (ii) and (iii) of Theorem 3 will also be proved for sets of types over a concept $C$ instead of sets of types over a knowledge base $\Sigma$. It will become clear that this does not make any essential difference.

The structure of this section is now as follows. We start with a proof of the (bounded) finite model property of concept satisfiability for both non-symmetric and symmetric cases. This result will first be used to prove Theorem 3 (iii). And then it will be employed as a basic ingredient for the proof of the upper bound of (i) for the non-symmetric and symmetric cases. The crucial idea in this proof is that satisfiable $\mathcal{S} \mathcal{L}$-concepts can be satisfied in 'abstract tree distance spaces,' and that satisfiability in these tree distance spaces can be reduced (exponentially) to the emptiness problem for tree automata with one complemented pair, which is known to be decidable in polynomial time [8]. Claim (ii) will then be a simple corollary of the proof of (i).

\subsection{The finite model property}

Since here our concern is $\mathcal{S} \mathcal{L}$-concept satisfiability, we relativise the definitions of the closure, type, etc. to concepts of the language $\mathcal{S} \mathcal{L}$.

So suppose an $\mathcal{S} \mathcal{L}$-concept $C$ is given. Then $\mathrm{P}(C)$ is the set of all rational numbers (parameters) occurring in $C$, and

$$
\mathrm{P}^{+}(C)=\left\{b=a_{1}+\cdots+a_{n} \mid a_{i} \in \mathrm{P}(C), b \leq \max \mathrm{P}(C)\right\} .
$$

Denote by $\operatorname{sub}(C)$ the set of all subconcepts of $C$, and by $\operatorname{cl}(C)$ the smallest set of concepts closed under the following rules:

- $\operatorname{cl}(C)$ contains $\operatorname{sub}(C)$ and is closed under taking single negation,

- if $D_{1} \leftleftarrows D_{2} \in \operatorname{cl}(C)$ then $\exists D_{1}, \exists D_{2} \in \operatorname{cl}(C)$,

- if $\exists<a D \in \operatorname{cl}(C)$ or $\exists \leq a D \in \operatorname{cl}(C)$ then $\exists D \in \operatorname{cl}(C)$,

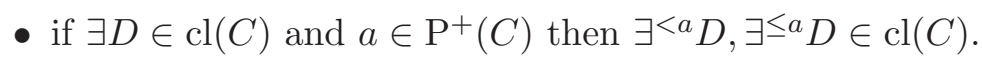

As we have already observed, the size of $\operatorname{cl}(C)$ is at most exponential in the length $l(C)$ of $C$ if the parameters are coded in binary.

If $\mathrm{P}^{+}(C)=\left\{a_{0}, \ldots, a_{N-1}\right\}$ with $a_{0}<\cdots<a_{N-1}$, then we set

$$
a_{N}=\min \left\{a+b \mid a, b \in \mathrm{P}^{+}(C), a+b>a_{N-1}\right\}, \quad \text { and } \overline{\mathrm{P}}(C)=\left\{a_{0}, \ldots, a_{N}\right\} .
$$

Given an $\mathcal{S} \mathcal{L}$-model $\mathcal{I}$ and its element $u$, define the type $T^{\mathcal{I}}(u)$ of $u$ in $\mathcal{I}$ by taking

$$
T^{\mathcal{I}}(u)=\left\{D \in \operatorname{cl}(C) \mid u \in D^{\mathcal{I}}\right\} .
$$

Theorem 4. Let $\mathcal{M}$ be the class of metric min-models or the class of min-models with (tr). If $C$ is satisfiable in $\mathcal{M}$ then $C$ is satisfiable in a finite model from $\mathcal{M}$ (whose size is at most exponential in the length of $C$ ).

Proof. The proof uses a more or less standard filtration argument from modal logic (see, e.g., [5]). The main difficulty will be to define a proper distance function on the filtrated model. 
We consider first the case of $\mathcal{M}$ containing arbitrary (not necessarily symmetric) minmodels with (tr). In fact, the technique we use below works for a wider class of models where (min) is relaxed to the following condition:

$$
d(x, Y)=\min \{d(x, y) \mid y \in Y\}
$$

(wmin)

for every point $x$ and every nonempty set $Y$. Such models are called weak min-models.

Non-symmetric case. Suppose that $C$ is satisfied in a weak min-model $\mathcal{I}$ with (tr). Our aim is to construct a finite (not necessarily symmetric) model $\overline{\mathcal{I}}$ with (tr) satisfying $C$.

Define an equivalence relation $\sim$ on $\Delta^{\mathcal{I}}$ by taking

$$
u \sim v \quad \text { iff } \quad T^{\mathcal{I}}(u)=T^{\mathcal{I}}(v),
$$

and let $[u]=\left\{v \in \Delta^{\mathcal{I}} \mid u \sim v\right\}$, for every $u \in \Delta$. The model $\overline{\mathcal{I}}$ will be based on the set $\Delta^{\overline{\mathcal{I}}}=\left\{[u] \mid u \in \Delta^{\mathcal{I}}\right\}$. To define a suitable distance function on $\Delta^{\overline{\mathcal{I}}}$, some extra work has to be done.

We first introduce two binary relations $R^{<a}$ and $R^{\leq a}$ on $\Delta^{\overline{\mathcal{I}}}$, for every $a \in \overline{\mathrm{P}}(C)$. Define $R^{<a_{N}}=R^{\leq a_{N}}=\Delta^{\overline{\mathcal{I}}} \times \Delta^{\overline{\mathcal{I}}}$ and, for $a \in \mathrm{P}^{+}(C)$, set $[u] R^{<a}[v]$ iff the following conditions are satisfied for all $\exists D \in \operatorname{cl}(C)$ and $b, a+b \in \mathrm{P}^{+}(C)$ :

$$
\begin{array}{rll}
v \in D^{\mathcal{I}} & \text { implies } & u \in\left(\exists^{<a} D\right)^{\mathcal{I}}, \\
v \in\left(\exists^{\leq b} D\right)^{\mathcal{I}} & \text { implies } & u \in\left(\exists^{<a+b} D\right)^{\mathcal{I}} .
\end{array}
$$

Let $d^{<}([u],[v])=\min \left\{a \in \overline{\mathrm{P}}(C) \mid[u] R^{<a}[v]\right\}$. It should be clear that these definitions do not depend on the choice of $u$ and $v$ from $[u]$ and $[v]$.

Similarly, for $a \in \mathrm{P}^{+}(C)$, we set $[u] R^{\leq a}[v]$ iff the following conditions are satisfied for all $\exists D \in \operatorname{cl}(C)$ and $b, a+b \in \mathrm{P}^{+}(C)$ :

$$
\begin{array}{rll}
v \in D^{\mathcal{I}} & \text { implies } & u \in\left(\exists^{\leq a} D\right)^{\mathcal{I}}, \\
v \in\left(\exists^{\leq b} D\right)^{\mathcal{I}} & \text { implies } & u \in\left(\exists^{\leq a+b} D\right)^{\mathcal{I}}, \\
v \in\left(\exists^{<b} D\right)^{\mathcal{I}} & \text { implies } & u \in\left(\exists^{<a+b} D\right)^{\mathcal{I}} .
\end{array}
$$

Define $d^{\leq}([u],[v])=\min \left\{a \in \overline{\mathrm{P}}(C) \mid[u] R^{\leq a}[v]\right\}$.

Let $a \oplus b=\min \left\{a_{N}, a+b\right\}$ for $a, b \in \overline{\mathrm{P}}(C)$. It is straightforward to check the following properties:

Lemma 5. For all $a, b \in \overline{\mathrm{P}}(C)$ and $u, v \in \Delta^{\mathcal{I}}$, we have:

(1) $R^{<a} \circ R^{\leq b} \subseteq R^{<a \oplus b}, R^{\leq a} \circ R^{<b} \subseteq R^{<a \oplus b}, R^{\leq a} \circ R^{\leq b} \subseteq R^{\leq a \oplus b}$,

(2) if $d(u, v)<$ a then $[u] R^{<a}[v]$ and $d^{<}([u],[v]) \leq a$,

if $d(u, v) \leq a$ then $[u] R^{\leq a}[v]$ and $d \leq([u],[v]) \leq a$,

$d \leq([u],[v]) \leq d^{<}([u],[v])$.

Proof. (1) Assume that $[u] R^{<a}[v]$ and $[v] R^{\leq b}[w]$. If $a \oplus b=a_{N}$, then $[u] R^{<a \oplus b}[w]$ always holds. Now suppose that $a \oplus b=a+b<a_{N}$. To prove that $[u] R^{<a \oplus b}[w]$, assume first that $w \in D^{\mathcal{I}}$ and $\exists D \in \operatorname{cl}(C)$. Then, by $(8), v \in\left(\exists^{\leq b} D\right)^{\mathcal{I}}$ and, by $(7), u \in\left(\exists^{<a+b} D\right)^{\mathcal{I}}$. Now 
assume that $w \in\left(\exists^{\leq c} D\right)^{\mathcal{I}}, \exists D \in \operatorname{cl}(C)$, and $c, c+a+b \in \mathrm{P}^{+}(C)$. Then, by $(8), v \in(\exists \leq c+b D)^{\mathcal{I}}$ and, by (7), $u \in\left(\exists^{<c+a+b} D\right)^{\mathcal{I}}$. The remaining two inclusions of (1) are proved similarly.

(2) Assume that $d(u, v)<a$. Then $u \in\left(\exists^{<a} D\right)^{\mathcal{I}}$ follows from $v \in D^{\mathcal{I}}$ by the truth condition for $\exists^{<a}$. Moreover, for the same reason $v \in\left(\exists^{\leq b} D\right)^{\mathcal{I}}$ implies $u \in\left(\exists^{<a} \exists \leq b D\right)^{\mathcal{I}}$. Hence, by the triangle inequality for $d, u \in\left(\exists^{<a+b} D\right)^{\mathcal{I}}$. We have shown that $[u] R^{<a}[v]$. Now $d^{<}([u],[v]) \leq a$ follows from the definition of the function $d^{<}$. The remaining claims of (2) are proved analogously.

Corollary 6. The functions $d^{<}$and $d^{\leq}$satisfy the triangle inequality. Moreover, for all $u, v, w \in \Delta^{\mathcal{I}}$, we have:

$$
\begin{aligned}
& d^{<}([w],[u]) \leq d^{<}([w],[v])+d^{\leq}([v],[u]), \\
& d^{<}([w],[u]) \leq d^{\leq}([w],[v])+d^{<}([v],[u]) .
\end{aligned}
$$

Proof. We only prove the triangle inequality for $d^{<}$. The remaining inequalities are proved in the same way. Suppose that $d^{<}([u],[v])=a$ and $d^{<}([v],[w])=b$. Then $[u] R^{<a}[v]$ and $[v] R^{<b}[w]$. Therefore $[v] R^{\leq b}[w]$. By Lemma $5(1),[u] R^{<a \oplus b}[w]$. Hence $d^{<}([u],[w]) \leq a \oplus b$. It follows that $d^{<}([u],[w]) \leq d^{<}([u],[v])+d^{<}([v],[w])$.

Thus, $d^{\leq}$and $d^{<}$can be regarded as approximations for the distance function on $\Delta^{\overline{\mathcal{I}}}$ we need in the following sense: $d^{\leq}([u],[v])=a$ (or $\left.d^{<}([u],[v])=b\right)$ means that the distance from $[u]$ to $[v]$ should be $\leq a$ (or $<b$, respectively). Moreover, given the amount of information we have, $d^{\leq}$and $d^{<}$are the best approximations of this sort.

Let $\overline{\mathrm{P}}(C)=\left\{a_{0}, \ldots, a_{N}\right\}$ and $a_{0}<\cdots<a_{N}$ as in (5). Then, having a strict inequality $d^{\leq}([u],[v])=a_{s}<d^{<}([u],[v])$, we can conclude that the distance from $[u]$ to $[v]$ is actually $a_{s}$. And if we actually have the equality $d^{\leq}([u],[v])=a_{s}=d^{<}([u],[v])$, then we can only conclude that the distance from $[u]$ to $[v]$ is strictly between $a_{s-1}$ and $a_{s}$. In this case we will define this distance as $a_{s}-e$, for some 'sufficiently small' $e$.

More precisely, for every $a_{s} \in \overline{\mathrm{P}}(C)$, we introduce a set $E_{s}$ of sufficiently small positive numbers that will serve as such adjusting values. To ensure that the triangle inequality still holds for the modified distances, we need to fulfil the following requirements:

$$
\begin{aligned}
& a_{r}<a_{s}+a_{t} \text { implies } a_{r}<a_{s}-e_{s}+a_{t}-e_{t}, \\
& a_{r}=a_{s}+a_{t} \text { implies } a_{r}-e_{r} \leq a_{s}-e_{s}+a_{t}-e_{t},
\end{aligned}
$$

for all $r, s, t \leq N$ and $e_{i} \in E_{i}$. This can be achieved as follows. Define $\delta$ and $\varepsilon_{0}, \ldots, \varepsilon_{N}$ by taking

$$
\begin{gathered}
\delta=\min \{a+b-c \mid a, b, c \in \overline{\mathrm{P}}(C) \cup\{0\}, a+b>c\} \\
\delta / 4=\varepsilon_{N}, \quad \varepsilon_{N} / 4=\varepsilon_{N-1}, \quad \ldots, \quad \varepsilon_{1} / 4=\varepsilon_{0} .
\end{gathered}
$$

Now, if $K=\left|\Delta^{\overline{\mathcal{I}}}\right|$ then we let $E_{s}$ be any (fixed) subset of the open interval $\left(\varepsilon_{s}, 2 \varepsilon_{s}\right)$ such that $\left|E_{s}\right|=K^{2}$. For all $s=0, \ldots, N$, define

$$
J_{s}=\left\{([u],[v]) \in \Delta^{\overline{\mathcal{I}}} \times \Delta^{\overline{\mathcal{I}}} \mid d^{\leq}([u],[v])=d^{<}([u],[v])=a_{s} \text { and }[u] \neq[v]\right\} .
$$

Note that $\left|J_{s}\right| \leq K^{2}$. For each $[u] \in \Delta^{\overline{\mathcal{I}}}$, fix some representative $\widehat{u} \in[u]$, and, for each $([w],[v]) \in J_{s}$, choose a number $\varepsilon([w],[v]) \in E_{s}$ in such a way that the condition

$$
\varepsilon([w],[v])>\varepsilon([w],[u]) \quad \text { iff } \quad d(\widehat{w},[v])<d(\widehat{w},[u])
$$


holds for all $([w],[v])$ and $([w],[u])$ in $J_{s}$.

We are now in a position to define a distance function $d^{\overline{\mathcal{I}}}$ on $\Delta^{\overline{\mathcal{I}}}$ : for all $[u],[v] \in \Delta^{\overline{\mathcal{I}}}$, let

- $d^{\overline{\mathcal{I}}}([u],[u])=0$,

- $d^{\overline{\mathcal{I}}}([u],[v])=d^{\leq}([u],[v]), \quad$ if $[u] \neq[v]$ and $d^{\leq}([u],[v])<d^{<}([u],[v])$,

- $d^{\bar{I}}([u],[v])=d^{\leq}([u],[v])-\varepsilon([u],[v]), \quad$ if $[u] \neq[v]$ and $d^{\leq}([u],[v])=d^{<}([u],[v])$.

Finally, we set $D^{\overline{\mathcal{I}}}=\left\{[u] \mid u \in D^{\mathcal{I}}\right\}$ and $\ell^{\overline{\mathcal{I}}}=\{\ell\}^{\mathcal{I}}$, for every concept name $D$ and every object name $\ell$.

Lemma 7. $\overline{\mathcal{I}}$ is an $\mathcal{S} \mathcal{L}$-model satisfying the triangle inequality.

Proof. We need to show that $d^{\overline{\mathcal{I}}}$ is a distance function satisfying $(\operatorname{tr})$. We have $d^{\overline{\mathcal{I}}}([u],[v])=0$ by definition. And if $[u] \neq[v]$ and $d^{\leq}([u],[v])=a_{s}$ then $d^{\overline{\mathcal{I}}}([u],[v]) \geq a_{s}-2 \varepsilon_{s}>\delta-\delta / 2>0$.

Now consider $[w],[v],[u] \in \Delta^{\overline{\mathcal{I}}}$. We may assume that $[w],[v],[u]$ are all different, since otherwise the triangle inequality trivially holds. Let $d^{\leq}([w],[v])=a_{s}, d^{\leq}([v],[u])=a_{t}$ and $d^{\leq}([w],[u])=a_{r}$. By Corollary $6, a_{r} \leq a_{s}+a_{t}$. If $a_{r}<a_{s}+a_{t}$ then $a_{s}+a_{t}-a_{r} \geq \delta$, $\varepsilon_{s}, \varepsilon_{t} \leq \delta / 4$, and therefore

$$
d^{\overline{\mathcal{I}}}([w],[v])+d^{\overline{\mathcal{I}}}([v],[u])-d^{\overline{\mathcal{I}}}([w],[u])>a_{s}+a_{t}-a_{r}-2 \varepsilon_{s}-2 \varepsilon_{t} \geq 0 .
$$

Suppose now that $a_{r}=a_{s}+a_{t}$. Then $a_{s}, a_{t}<a_{r}$, and hence $\varepsilon_{s}, \varepsilon_{t} \leq \varepsilon_{r} / 4$. Therefore, if $d^{<}([w],[u])=a_{r}$, then

$$
d^{\overline{\mathcal{I}}}([w],[v])+d^{\overline{\mathcal{I}}}([v],[u])-d^{\overline{\mathcal{I}}}([w],[u])>-2 \varepsilon_{s}-2 \varepsilon_{t}+\varepsilon_{r} \geq 0 .
$$

Thus, it remains to consider the case $d^{<}([w],[u])>a_{r}$. But then, by Corollary 6 , we have

$$
\begin{aligned}
& d^{<}([w],[v]) \geq d^{<}([w],[u])-d^{\leq}([v],[u])>a_{r}-a_{t}=a_{s}, \\
& d^{<}([v],[u]) \geq d^{<}([w],[u])-d^{\leq}([w],[v])>a_{r}-a_{s}=a_{t} .
\end{aligned}
$$

Therefore, $d^{\overline{\mathcal{I}}}([w],[u])=a_{r}, d^{\overline{\mathcal{I}}}([w],[v])=a_{s}$, and $d^{\overline{\mathcal{I}}}([v],[u])=a_{t}$, i.e., the triangle inequality holds.

Thus, to complete the proof of Theorem 4 for the non-symmetric case, it is enough to show the following:

Lemma 8. For all $w \in \Delta^{\mathcal{I}}$ and $D \in \operatorname{cl}(C)$, we have $[w] \in D^{\overline{\mathcal{I}}}$ iff $w \in D^{\mathcal{I}}$.

Proof. The proof is by induction on the construction of $D$. The basis of induction and the Boolean cases are trivial.

Case $D=D_{0} \leftleftarrows D_{1}$. We can assume that $D_{0}^{\mathcal{I}}, D_{1}^{\mathcal{I}} \neq \emptyset$, since otherwise the claim follows easily from the induction hypothesis. Let $w \in\left(D_{0} \leftleftarrows D_{1}\right)^{\mathcal{I}}$. Then $d^{\mathcal{I}}\left(\widehat{w}, D_{0}^{\mathcal{I}}\right)<d^{\mathcal{I}}\left(\widehat{w}, D_{1}^{\mathcal{I}}\right)$ where $\widehat{w}$ is the fixed representative of $[w]$. There exist $v_{0} \in D_{0}^{\mathcal{I}}$ and $\left[v_{1}\right] \in D_{1}^{\overline{\mathcal{I}}}$ such that $d^{\mathcal{I}}\left(\widehat{w}, v_{0}\right)=d^{\mathcal{I}}\left(\widehat{w}, D_{0}^{\mathcal{I}}\right)$ and $d^{\overline{\mathcal{I}}}\left([w],\left[v_{1}\right]\right)=d^{\overline{\mathcal{I}}}\left([w], D_{1}^{\overline{\mathcal{I}}}\right)$ (note that by the induction hypothesis we also have $\left[v_{0}\right] \in D_{0}^{\overline{\mathcal{I}}}$ and $\left.v_{1} \in D_{1}^{\mathcal{I}}\right)$. We thus need to show that $d^{\overline{\mathcal{I}}}\left([w],\left[v_{0}\right]\right)<d^{\overline{\mathcal{I}}}\left([w],\left[v_{1}\right]\right)$. First, note that $d^{\mathcal{I}}\left(\widehat{w},\left[v_{0}\right]\right)=d^{\mathcal{I}}\left(\widehat{w}, D_{0}^{\mathcal{I}}\right)<d^{\mathcal{I}}\left(\widehat{w}, D_{1}^{\mathcal{I}}\right) \leq d^{\mathcal{I}}\left(\widehat{w},\left[v_{1}\right]\right)$. Next, we prove that $d^{<}\left([w],\left[v_{0}\right]\right) \leq d^{\leq}\left([w],\left[v_{1}\right]\right)$. Let $d^{\leq}\left([w],\left[v_{1}\right]\right)=a$, so we may assume that $a<a_{N}$, because 
otherwise the statement is trivial. We have $[w] \in R^{\leq a}\left[v_{1}\right]$ and $\exists^{<a} D_{0}, \exists^{\leq a} D_{1} \in \operatorname{cl}(C)$, since $D_{0} \leftleftarrows D_{1} \in \operatorname{cl}(C)$. Therefore $v_{1} \in D_{1}^{\mathcal{I}}$ implies $\widehat{w} \in\left(\exists^{\leq a} D_{1}\right)^{\mathcal{I}}$, whence $\widehat{w} \in\left(D_{0} \leftleftarrows D_{1}\right)^{\mathcal{I}}$ implies $\widehat{w} \in\left(\exists^{<a} D_{0}\right)^{\mathcal{I}}$. Thus $d^{\mathcal{I}}\left(\widehat{w}, v_{0}\right) \leq d^{\mathcal{I}}\left(\widehat{w}, D_{0}^{\mathcal{I}}\right)<a$, and, by Lemma $5(2), d^{<}\left([w],\left[v_{0}\right]\right) \leq a$, as required.

We now have three subcases. If $d \leq\left([w],\left[v_{1}\right]\right)<d^{<}\left([w],\left[v_{1}\right]\right)$, then by definition we have $d^{\overline{\mathcal{I}}}\left([w],\left[v_{0}\right]\right)<d^{<}\left([w],\left[v_{0}\right]\right)<d^{\leq}\left([w],\left[v_{1}\right]\right)=d^{\overline{\mathcal{I}}}\left([w],\left[v_{1}\right]\right)$. If $d^{\leq}\left([w],\left[v_{0}\right]\right)<d^{<}\left([w],\left[v_{0}\right]\right)$ then $d^{\leq}\left([w],\left[v_{1}\right]\right)-d^{\leq}\left([w],\left[v_{0}\right]\right) \geq \delta, \varepsilon\left([w],\left[v_{1}\right]\right)<\delta / 2$, and therefore

$$
d^{\overline{\mathcal{I}}}\left([w],\left[v_{1}\right]\right)-d^{\overline{\mathcal{I}}}\left([w],\left[v_{0}\right]\right) \geq d^{\leq}\left([w],\left[v_{1}\right]\right)-\varepsilon\left([w],\left[v_{1}\right]\right)-d^{\leq}\left([w],\left[v_{0}\right]\right)>0 .
$$

Finally, if $d^{\leq}\left([w],\left[v_{0}\right]\right)=d^{<}\left([w],\left[v_{0}\right]\right)$ and $d^{\leq}([w],[v])=d^{<}([w],[v])$ then

$$
d^{\overline{\mathcal{I}}}\left([w],\left[v_{1}\right]\right)-d^{\overline{\mathcal{I}}}\left([w],\left[v_{0}\right]\right)=-\varepsilon\left([w],\left[v_{1}\right]\right)+\varepsilon\left([w],\left[v_{0}\right]\right)>0,
$$

since $d^{\mathcal{I}}\left(\widehat{w},\left[v_{0}\right]\right)<d^{\mathcal{I}}\left(\widehat{w},\left[v_{1}\right]\right)$.

In the same way one can show that $w \notin\left(D_{0} \leftleftarrows D_{1}\right)^{\mathcal{I}}$ implies $d^{\overline{\mathcal{I}}}\left([w], D_{1}^{\overline{\mathcal{I}}}\right) \leq d^{\overline{\mathcal{I}}}\left([w], D_{0}^{\overline{\mathcal{I}}}\right)$.

Case $D=\exists^{<a} D_{0}$. Let $w \in\left(\exists^{<a} D_{0}\right)^{\mathcal{I}}$, i.e., there is $v \in D_{0}^{\mathcal{I}}$ with $d^{\mathcal{I}}(w, v)<a$. Then $d^{\overline{\mathcal{I}}}([w],[v])<d^{<}([w],[v]) \leq a$ in view of Lemma $5(2)$, and $[v] \in D_{0}^{\overline{\mathcal{I}}}$ by the induction hypothesis.

Conversely, suppose that $[v] \in D_{0}^{\overline{\mathcal{I}}}$ for some $[v]$ with $d^{\overline{\mathcal{I}}}([w],[v])<a$. Then $d \leq([w],[v]) \leq a$, and $v \in D_{0}^{\mathcal{I}}$ by the induction hypothesis. If $d^{\leq}([w],[v])=d^{<}([w],[v])$, then $d^{<}([w],[v]) \leq a$, $w R^{<a} v$ and, therefore, $w \in\left(\exists^{<a} D_{0}\right)^{\mathcal{I}}$, since $v \in D_{0}^{\mathcal{I}}$. And if $d^{\leq}([w],[v])<d^{<}([w],[v])$, then $d^{\leq}([w],[v])=d^{\overline{\mathcal{I}}}([w],[v])<a$. Let $d^{\leq}([w],[v])=b$. Then $[w] R^{\leq b}[v]$ and, since $v \in D_{0}^{\mathcal{I}}$, we have $w \in\left(\exists^{\leq b} D_{0}\right)^{\mathcal{I}} \subseteq\left(\exists^{<a} D_{0}\right)^{\mathcal{I}}$.

Case $D=\exists^{\leq a} D_{0}$. Let $w \in\left(\exists^{\leq a} D_{0}\right)^{\mathcal{I}}$, i.e., there is $v \in D_{0}^{\mathcal{I}}$ with $d^{\mathcal{I}}(w, v) \leq a$. Then $d^{\overline{\mathcal{I}}}([w],[v]) \leq d^{\leq}([w],[v]) \leq a$ in view of Lemma $5(2)$; and $[v] \in D_{0}^{\overline{\mathcal{I}}}$ by the induction hypothesis. Conversely, suppose $[v] \in D_{0}^{\overline{\mathcal{I}}}$ for some $[v]$ with $d^{\overline{\mathcal{I}}}([w],[v]) \leq a$. Then $d^{\leq}([w],[v]) \leq a$, i.e., $[w] R^{\leq a}[v]$, and $v \in D_{0}^{\mathcal{I}}$ by the induction hypothesis. Therefore, $\left.w \in(\exists \leq a) D_{0}\right)^{\mathcal{I}}$.

Symmetric case. Now let $\mathcal{I}$ be a metric min-model. We first note one important property that follows from condition (min). For every $A, B, C \subseteq \Delta^{\mathcal{I}}, A \neq \emptyset$, we have

$$
\text { if } \forall a \in A d^{\mathcal{I}}(a, B)<d^{\mathcal{I}}(a, C) \text { then } d^{\mathcal{I}}(A, B)<d^{\mathcal{I}}(A, C) .
$$

Indeed, let $d^{\mathcal{I}}(A, B)=d^{\mathcal{I}}\left(a_{0}, B\right)$ and $d^{\mathcal{I}}(A, C)=d^{\mathcal{I}}\left(a_{1}, C\right)$ for some $a_{0}, a_{1} \in A$. Then $d^{\mathcal{I}}\left(a_{0}, B\right) \leq d^{\mathcal{I}}\left(a_{1}, B\right)<d^{\mathcal{I}}\left(a_{1}, C\right)$.

As in the previous case, to construct a finite model $\overline{\mathcal{I}}$ from $\mathcal{I}$ we first introduce the equivalence relation $\sim$ on $\Delta^{\mathcal{I}}$ by $(6)$, denote by $[u]$ the equivalence class of $u \in \Delta^{\mathcal{I}}$, and set $\Delta^{\overline{\mathcal{I}}}=\left\{[u] \mid u \in \Delta^{\mathcal{I}}\right\}$. For every $a \in \overline{\mathrm{P}}(C)$, define relations $R^{<a}$ and $R^{\leq a}$ by (7) and (8). Let $S^{<a}=R^{<a} \cap\left(R^{<a}\right)^{-1}$ and $S^{\leq a}=R^{\leq a} \cap\left(R^{\leq a}\right)^{-1}$. Now we set

$$
\begin{aligned}
& d^{<}([u],[v])=\min \left\{a \in \overline{\mathrm{P}}(C) \mid[u] S^{<a}[v]\right\}, \\
& d^{\leq}([u],[v])=\min \left\{a \in \overline{\mathrm{P}}(C) \mid[u] S^{\leq a}[v]\right\} .
\end{aligned}
$$

As an easy corollary of Lemma 5 we obtain

Lemma 9. For all $a, b \in \overline{\mathrm{P}}(C)$ and all $u, v \in \Delta^{\mathcal{I}}$, we have 
(1) $S^{<a} \circ S^{\leq b} \subseteq S^{<a \oplus b}, \quad S^{\leq a} \circ S^{<b} \subseteq S^{<a \oplus b}, \quad S^{\leq a} \circ S^{\leq b} \subseteq S^{\leq a \oplus b}$,

(2) if $d(u, v)<a$ then $[u] S^{<a}[v]$ and $d^{<}([u],[v]) \leq a$,

if $d(u, v) \leq a$ then $[u] S \leq a[v]$ and $d \leq([u],[v]) \leq a$,

$d \leq([u],[v]) \leq d^{<}([u],[v])$.

Corollary 10. The functions $d^{<}$and $d \leq$ are symmetric and satisfy the triangle inequality.

As in the previous case, we introduce $\delta, \varepsilon_{s}, E_{s}$, and $J_{s}$; see (9)-(10). Now, for every $s \leq N$ and $([w],[v]) \in J_{s}$, we choose $\varepsilon([w],[v])$ in $E_{s}$ in such a way that the condition

$$
\varepsilon([w],[v])<\varepsilon\left(\left[w^{\prime}\right],\left[v^{\prime}\right]\right) \quad \text { iff } \quad d([w],[v])>d\left(\left[w^{\prime}\right],\left[v^{\prime}\right]\right)
$$

holds for all $([w],[v])$ and $\left(\left[w^{\prime}\right],\left[v^{\prime}\right]\right)$ in $J_{s}$. Again, this is possible because $\left|J_{s}\right| \leq\left|E_{s}\right|$. Note that (12) guarantees that $\varepsilon$ is a symmetric function.

We are now in a position to define the metric function $d^{\overline{\mathcal{I}}}$ : for $[u],[v] \in \Delta^{\overline{\mathcal{I}}}$,

- $d^{\overline{\mathcal{I}}}([u],[u])=0$,

- $d^{\overline{\mathcal{I}}}([u],[v])=d^{\leq}([u],[v]), \quad$ if $[u] \neq[v]$ and $d^{\leq}([u],[v])<d^{<}([u],[v])$,

- $d^{\overline{\mathcal{I}}}([u],[v])=d^{\leq}([u],[v])-\varepsilon([u],[v]), \quad$ if $[u] \neq[v]$ and $d^{\leq}([u],[v])=d^{<}([u],[v])$.

Finally, we set $D^{\overline{\mathcal{I}}}=\left\{[u] \mid u \in D^{\mathcal{I}}\right\}$ and $\ell^{\overline{\mathcal{I}}}=\{\ell\}^{\mathcal{I}}$ for every concept name $D$ and every object name $\ell$.

Lemma 11. $\overline{\mathcal{I}}$ is a metric model.

Proof. The proof is almost the same as the proof of Lemma 7. The only difference is the symmetry condition, where it is enough to observe that the functions $d^{<}, d^{\leq}$and $d$ are symmetric, whence $\varepsilon$ is symmetric, and so is $d^{\overline{\mathcal{L}}}$.

Lemma 12. For all $w \in \Delta^{\mathcal{I}}$ and $D \in \operatorname{cl}(C)$, we have $[w] \in D^{\overline{\mathcal{I}}}$ iff $w \in D^{\mathcal{I}}$.

Proof. The proof (by induction on the construction of $D$ ) is similar to that of Lemma 8 . The only essential difference is that here we use property (11) instead of picking up representatives in each class $[w]$.

Case $D=D_{0} \leftleftarrows D_{1}$. We assume that $D_{0}^{\mathcal{I}} \neq \emptyset$ and $D_{1}^{\mathcal{I}} \neq \emptyset$; otherwise the lemma follows easily from the induction hypothesis. Let $w \in\left(D_{0} \leftleftarrows D_{1}\right)^{\mathcal{I}}$. Then $d^{\mathcal{I}}\left(w^{\prime}, D_{0}^{\mathcal{I}}\right)<d^{\mathcal{I}}\left(w^{\prime}, D_{1}^{\mathcal{I}}\right)$ for every $w^{\prime} \in[w]$, and so $d^{\mathcal{I}}\left([w], D_{0}^{\mathcal{I}}\right)<d^{\mathcal{I}}\left([w], D_{1}^{\mathcal{I}}\right)$ in view of (11). There exist $w_{0} \in[w]$, $v_{0} \in D_{0}^{\mathcal{I}}$ and $\left[v_{1}\right] \in D_{1}^{\overline{\mathcal{I}}}$ such that $d^{\mathcal{I}}\left(w_{0}, v_{0}\right)=d^{\mathcal{I}}\left([w], D_{0}^{\mathcal{I}}\right)$ and $d^{\overline{\mathcal{I}}}\left([w],\left[v_{1}\right]\right)=d^{\overline{\mathcal{I}}}\left([w], D_{1}^{\overline{\mathcal{I}}}\right)$. By the induction hypothesis, $\left[v_{0}\right] \in D_{0}^{\overline{\mathcal{I}}}$ and $v_{1} \in D_{1}^{\mathcal{I}}$. So we need to show that $d^{\overline{\mathcal{I}}}\left([w],\left[v_{0}\right]\right)<$ $d^{\overline{\mathcal{I}}}\left([w],\left[v_{1}\right]\right)$. Notice first that $d^{\mathcal{I}}\left([w],\left[v_{0}\right]\right)=d^{\mathcal{I}}\left([w], D_{0}^{\mathcal{I}}\right)<d^{\mathcal{I}}\left([w], D_{1}^{\mathcal{I}}\right) \leq d^{\mathcal{I}}\left([w],\left[v_{1}\right]\right)$. Next we prove $d^{<}\left([w],\left[v_{0}\right]\right) \leq d^{\leq}\left([w],\left[v_{1}\right]\right)$. Let $d^{\leq}\left([w],\left[v_{1}\right]\right)=a$. We may assume that $a<a_{N}$

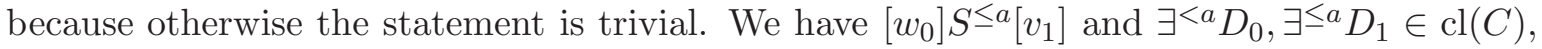
since $D_{0} \leftleftarrows D_{1} \in \operatorname{cl}(C)$. Then $v_{1} \in D_{1}^{\mathcal{I}}$ implies $w_{0} \in\left(\exists^{\leq a} D_{1}\right)^{\mathcal{I}}$, and so $w_{0} \in\left(D_{0} \leftleftarrows\right.$ $\left.D_{1}\right)^{\mathcal{I}}$ implies $w_{0} \in\left(\exists^{<a} D_{0}\right)^{\mathcal{I}}$. Thus $d^{\mathcal{I}}\left(w_{0}, v_{0}\right) \leq d^{\mathcal{I}}\left(w_{0}, D_{0}^{\mathcal{I}}\right)<a$, and, by Lemma $9(2)$, $d^{<}\left([w],\left[v_{0}\right]\right) \leq a$, as required.

Consider three subcases. If $d^{\leq}\left([w],\left[v_{1}\right]\right)<d^{<}\left([w],\left[v_{1}\right]\right)$, then by definition we have $d^{\overline{\mathcal{I}}}\left([w],\left[v_{0}\right]\right)<d^{<}\left([w],\left[v_{0}\right]\right) \leq d^{\leq}\left([w],\left[v_{1}\right]\right)=d^{\overline{\mathcal{I}}}\left([w],\left[v_{1}\right]\right)$. 
If $d^{\leq}\left([w],\left[v_{0}\right]\right)<d^{<}\left([w],\left[v_{0}\right]\right)$ then $d^{\leq}\left([w],\left[v_{1}\right]\right)-d^{\leq}\left([w],\left[v_{0}\right]\right) \geq \delta, \varepsilon\left([w],\left[v_{1}\right]\right)<\delta / 2$ and, therefore,

$$
d^{\overline{\mathcal{I}}}\left([w],\left[v_{1}\right]\right)-d^{\overline{\mathcal{I}}}\left([w],\left[v_{0}\right]\right) \geq d^{\leq}\left([w],\left[v_{1}\right]\right)-\varepsilon\left([w],\left[v_{1}\right]\right)-d^{\leq}\left([w],\left[v_{0}\right]\right)>0 .
$$

Finally, if $d^{\leq}\left([w],\left[v_{0}\right]\right)=d^{<}\left([w],\left[v_{0}\right]\right)$ and $d^{\leq}([w],[v])=d^{<}([w],[v])$ then

$$
d^{\overline{\mathcal{I}}}\left([w],\left[v_{1}\right]\right)-d^{\overline{\mathcal{I}}}\left([w],\left[v_{0}\right]\right)=-\varepsilon\left([w],\left[v_{1}\right]\right)+\varepsilon\left([w],\left[v_{0}\right]\right)>0,
$$

since $d^{\mathcal{I}}\left([w],\left[v_{0}\right]\right)<d^{\mathcal{I}}\left([w],\left[v_{1}\right]\right)$. Similarly, starting from $w \notin\left(D_{0} \leftleftarrows D_{1}\right)^{\mathcal{I}}$, we obtain $d^{\overline{\mathcal{I}}}\left([w], D_{1}^{\overline{\mathcal{I}}}\right) \leq d^{\overline{\mathcal{I}}}\left([w], D_{0}^{\overline{\mathcal{I}}}\right)$.

The remaining part of the proof is similar to that of Lemma 8.

This completes the proof of Theorem 4 .

Remark 13. Theorem 4 and its proof give a nondeterministic exponential time algorithm for checking satisfiability of a given concept $C$. Indeed, as we have already observed, the size of $\overline{\mathrm{P}}(C)$ and $\operatorname{cl}(C)$ is exponential in $l(C)$. Moreover, the number of different types is also exponential in $l(C)$, since every type $T$ is determined by the following:

- for every subterm $D$ of $C$, whether $D$ belongs to $T$,

- for every subterm of $C$ of the form $\exists D$, what is a minimal value $b$ (if any) such that $\exists^{\leq b} D$ belongs to $T$, and whether $\exists^{<b} D$ belongs to $T$.

Therefore, if $C$ is satisfied in a class of models $\mathcal{M}$ under consideration, then $C$ is satisfied in a finite model in $\mathcal{M}$ of exponential in $l(C)$ size. Moreover, the distance function of this model can possess the values from a prescribed set of exponential in $l(C)$ size. So the existence of such a model can be detected nondeterministically in exponential in $l(C)$ time.

\subsection{Proof of Theorem 3 (iii)}

Fix an $\mathcal{S} \mathcal{L}$-concept $C$. First we relativise the general definition of a type over a $\mathrm{KB} \Sigma$ to the case of a single concept. Namely, we say that a subset $T$ of $\operatorname{cl}(C)$ is a type for $C$ if it satisfies the following conditions:

- $D_{1} \sqcap D_{2} \in T$ iff $D_{1}, D_{2} \in T$, for every $D_{1} \sqcap D_{2} \in \operatorname{cl}(C)$,

- $\neg D \in T$ iff $D \notin T$, for every $\neg D \in \operatorname{cl}(C)$,

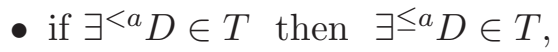

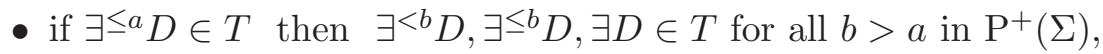

- if $D_{1} \leftleftarrows D_{2} \in T$ and $\exists \leq a D_{2} \in T$ then $\exists^{<a} D_{1} \in T$,

(The last condition of the definition of types over $\Sigma$ is irrelevant in this case because all models now are based on a single similarity space.) As we observed above, the number of types for $C$ is exponential in $l(C)$.

Let $\mathcal{T}$ be a realisable set of types, let $T_{1}, \ldots, T_{n}$ be an enumeration of those of them that do not contain nominals, and let $T_{n+1}, \ldots, T_{m}$ be the remaining types in $\mathcal{T}$. As we saw in the proof of Lemma 4, if $\mathcal{T}$ is realised in some model, then it is also realised in a (finite) model $\mathcal{I}$ such that $T^{\mathcal{I}}(u) \neq T^{\mathcal{I}}(v)$ whenever $u \neq v$. In other words, every type from $\mathcal{T}$ is realised by precisely one point from $\mathcal{I}$. Denote by $u_{i}$ the point realising $T_{i}, i=1, \ldots, m$. 
Now let $k_{1}, \ldots, k_{n}$ with $k_{i} \in \mathbb{N}^{>0} \cup\{\omega\}$ be given, and let $k_{j}=1$ for $j=n+1, \ldots, m$. Our aim is to construct, starting from $\mathcal{I}$, a model $\mathcal{J}$ such that $\left|T_{1}^{\mathcal{J}}\right|=k_{1}, \ldots,\left|T_{m}^{\mathcal{J}}\right|=k_{m}$. Let $U_{i}$ be a set of cardinality $k_{i}-1$. We assume that all of the $U_{i}$ and $\Delta^{\mathcal{I}}$ are pairwise disjoint. Let $U_{i}^{+}=U_{i} \cup\left\{u_{i}\right\}$ and let $d=\min \left\{d^{\mathcal{I}}(u, v) \mid u, v \in \Delta^{\mathcal{I}}, u \neq v\right\}$. Construct a new model $\mathcal{J}$ by taking

- $\Delta^{\mathcal{J}}=U_{1}^{+} \cup \cdots \cup U_{m}^{+}$,

- $d^{\mathcal{J}}(u, v)=d^{\mathcal{I}}\left(u_{i}, u_{j}\right)$ for $u \in U_{i}^{+}, v \in U_{j}^{+}, i \neq j$,

- $d^{\mathcal{J}}(u, v)=d$ for $u, v \in U_{i}, u \neq v$,

- $\ell^{\mathcal{J}}=\ell^{\mathcal{I}}$, for every object name $\ell$,

- $u \in A^{\mathcal{J}}$ iff $u \in U_{i}^{+}$and $u_{i} \in A^{\mathcal{I}}$, for every concept name $A$.

It is readily checked by induction that the model $\mathcal{J}$ is as required.

\subsection{Proof of Theorem 3 (i)}

Now we show that the concept satisfiability problem for $\mathcal{S} \mathcal{L}$ is decidable in ExpTime for the binary coding of parameters. Fix again an arbitrary $\mathcal{S} \mathcal{L}$-concept $C$.

For each $a \in \overline{\mathrm{P}}(C)$, we introduce a fresh symbol $a^{-}$and set

$$
\mathrm{Q}(C)=\mathrm{P}^{+}(C) \cup\left\{a^{-} \mid a \in \overline{\mathrm{P}}(C)\right\} \cup\{0\}
$$

(note that $\mathrm{Q}(C)$ contains $a_{N}^{-}$, but not $a_{N}$ ). Define a linear order $<$ on $\mathrm{Q}(C)$ in such a way that 0 is its minimal element, and $b<a^{-}<a<a_{N}^{-}$whenever $b<a$ in $\mathrm{P}^{+}(C)$.

We are now in a position to introduce the 'building material' for constructing models from types.

A bouquet for $C$ is a triple $\mathfrak{B}=\left(\mathcal{T}_{\mathfrak{B}}, \leq_{\mathfrak{B}}, d_{\mathfrak{B}}\right)$ where

- $\mathcal{T}_{\mathfrak{B}}$ is a set of types for $C$ such that $2 \leq\left|\mathcal{T}_{\mathfrak{B}}\right| \leq l(C)$,

- $\leq_{\mathfrak{B}}$ is a linear quasi-order on $\mathcal{T}_{\mathfrak{B}}$ with a unique minimal element $T_{\mathfrak{B}} \in \mathcal{T}_{\mathfrak{B}}$, and

- $d_{\mathfrak{B}}$ is a map from $\mathcal{T}_{\mathfrak{B}}$ to $\mathrm{Q}(C)$,

and the following conditions are satisfied, for all $T, T^{\prime} \in \mathcal{T}_{\mathfrak{B}}$ :

(B1) $d_{\mathfrak{B}}(T)=0$ iff $T=T_{\mathfrak{B}}$,

(B2) $T \leq_{\mathfrak{B}} T^{\prime}$ implies $d_{\mathfrak{B}}(T) \leq d_{\mathfrak{B}}\left(T^{\prime}\right)$,

(B3) $d_{\mathfrak{B}}(T)=d_{\mathfrak{B}}\left(T^{\prime}\right) \in \mathrm{P}^{+}(C)$ implies $T \leq_{\mathfrak{B}} T^{\prime} \leq_{\mathfrak{B}} T$,

(B4) $D_{1} \leftleftarrows D_{2} \in T_{\mathfrak{B}}$ iff $D_{1} \leftleftarrows D_{2} \in \operatorname{cl}(C)$, there is $T \in \mathcal{T}_{\mathfrak{B}}$ for which $D_{1} \in T$, and $D_{2} \notin T^{\prime}$ whenever $T^{\prime} \leq_{\mathfrak{B}} T$,

(B5) $\exists^{<a} D \in T_{\mathfrak{B}}$ iff $\exists^{<a} D \in \operatorname{cl}(C)$ and there is $T \in \mathcal{T}_{\mathfrak{B}}$ such that $d_{\mathfrak{B}}(T) \leq a^{-}$and $D \in T$,

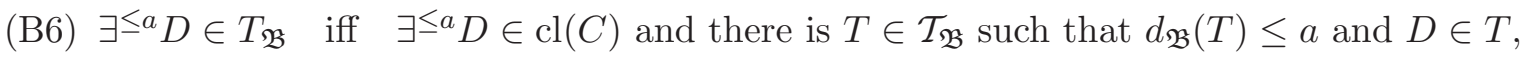

(B7) $\exists D \in T_{\mathfrak{B}}$ iff $\exists D \in T$,

(B8) if $a, b, a+b \in \mathrm{P}^{+}(C)$ and $\exists D \in \operatorname{cl}(C)$, then

$\exists^{<a} D \in T$ and $d_{\mathfrak{B}}(T) \leq b$ imply $\exists^{<a+b} D \in T_{\mathfrak{B}}$,

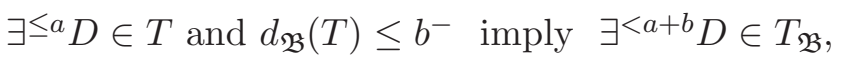

$\exists^{\leq a} D \in T$ and $d_{\mathfrak{B}}(T) \leq b$ imply $\exists^{\leq a+b} D \in T_{\mathfrak{B}}$. 
We will write $T \simeq_{\mathfrak{B}} T^{\prime}$ for $T \leq_{\mathfrak{B}} T^{\prime} \leq_{\mathfrak{B}} T$, and $T<_{\mathfrak{B}} T^{\prime}$ for $T \leq_{\mathfrak{B}} T^{\prime} \leq_{\mathfrak{B}} T$.

We use the notion of a bouquet to encode the local requirements ensuring that the type $T_{\mathfrak{B}}$ can be realised. A 'typical' bouquet is constructed as follows.

Let $\mathcal{I}$ be a model and $w \in \Delta^{\mathcal{I}}$. For every $\exists D \in T^{\mathcal{I}}(w)$ with $D \notin T^{\mathcal{I}}(w)$, choose a point $v \in D^{\mathcal{I}}$ with a minimal distance $d^{\mathcal{I}}(w, v)$; let $V$ denote the set of all selected points. We have $|V|<l(C)$, since $D \in \operatorname{sub}(C)$, for every $\exists D \in \operatorname{cl}(C)$; and we clearly can assume that $T^{\mathcal{I}}\left(v_{1}\right) \neq T^{\mathcal{I}}\left(v_{2}\right)$ for any distinct $v_{1}, v_{2} \in V$. If $|V| \geq 1$, then we define the bouquet $\left(\mathcal{T}_{V}^{\mathcal{I}}(w), \leq_{w}, d_{w}\right)$ induced by $w$ and $V$ in $\mathcal{I}$ by taking:

$$
\begin{aligned}
& \mathcal{T}_{V}^{\mathcal{I}}(w)=\left\{T^{\mathcal{I}}(w)\right\} \cup\left\{T^{\mathcal{I}}(v) \mid v \in V\right\}, \\
& T^{\mathcal{I}}\left(v_{1}\right) \leq_{w} T^{\mathcal{I}}\left(v_{2}\right) \text { iff } d^{\mathcal{I}}\left(w, v_{1}\right) \leq d^{\mathcal{I}}\left(w, v_{2}\right), \\
& d_{w}\left(T^{\mathcal{I}}(v)\right)= \begin{cases}a, & \text { if } d^{\mathcal{I}}(w, v)=a \in \mathrm{P}^{+}(C), \\
a^{-}, & \text {if } d^{\mathcal{I}}(w, v)<a=\min \left\{b \in \mathrm{P}^{+}(C) \mid d^{\mathcal{I}}(w, v) \leq b\right\}, \\
a_{N}^{-}, & \text {if } d^{\mathcal{I}}(w, v)>a_{N-1} .\end{cases}
\end{aligned}
$$

Note that if we require a certain type $T$ realised in $\mathcal{I}$ to be a member

of the bouquet then we can add to $V$ a point $v$ such that $d(w, v)$ is minimal with $T=T^{\mathcal{I}}(v)$ and form the bouquet induced by $w$ and $V \cup\{v\}$. In particular, if $\mathcal{I}$ realises at least two distinct types, then we can always find a set $V$ such that $w$ and $V$ induce a bouquet.

Note also that the number of all bouquets is exponential in $l(C)$.

In what follows we will only be working with models satisfying at least two distinct types, because checking satisfiability of a concept in a model satisfying only one type reduces to the case of pure Boolean concepts, which is decidable in NP.

Again we consider two cases.

Non-symmetric case. Let $\mathcal{N}$ be the set of nominals occurring in $C$. A set $B$ of bouquets is said to be nominal ready if there is a set $\left\{T_{\ell} \mid \ell \in \mathcal{N}\right\}$ of types such that

$$
B=\left\{\mathfrak{B} \mid \mathfrak{B} \text { is a bouquet, }\{\ell\} \in T \in \mathcal{T}_{\mathfrak{B}} \text { implies } T=T_{\ell}\right\} .
$$

Thus, every nominal ready set is determined by a certain set $\left\{T_{\ell} \mid \ell \in \mathcal{N}\right\}$ of the size $<l(C)$, hence there exist only exponentially many in $l(C)$ nominal ready sets.

Let $k=l(C)$. As usual, $\{1, \ldots, k\}^{*}$ denotes the set of all finite sequences of elements of $\{1, \ldots, k\}$. By the full $k$-ary tree over $\{1, \ldots, k\}^{*}$ we mean the tree that contains the empty sequence $\epsilon$ as its root, and the immediate successors (children) of each node $\alpha$ are precisely the nodes $\alpha i$, where $1 \leq i \leq k$. Given some set $L$ (of labels), a function $K:\{1, \ldots, k\}^{*} \rightarrow L$ will be called an $L$-labelled tree over $\{1, \ldots, k\}^{*}$.

A Hintikka tree satisfying $C$ is a $B$-labelled tree $K$ over $\{1, \ldots, k\}^{*}$, for some nominal ready set $B$ of bouquets, such that the following conditions are satisfied (where, as before, $T_{K(\alpha)}$ denotes the unique $\leq_{K(\alpha)}$-minimal element of the set of types $\mathcal{T}_{K(\alpha)}$ in the bouquet $K(\alpha))$ :

(H1) $C \in T_{K(\epsilon)}$,

(H2) for every nominal $\ell \in N$, there exists a type in $K(\epsilon)$ containing $\{\ell\}$,

(H3) for every $\alpha \in\{1, \ldots, k\}^{*}, K(\alpha)$ is a bouquet such that

$$
\mathcal{T}_{K(\alpha)} \backslash\left\{T_{K(\alpha)}\right\}=\left\{T_{K(\alpha i)} \mid 1 \leq i \leq k\right\},
$$

and $T_{K(\alpha)} \in \mathcal{T}_{K(\alpha i)}$, for all $1 \leq i \leq k$. 
Theorem 14. For every concept $C$, the following conditions are equivalent:

(a) $C$ is satisfiable in a min-model with (tr) and at least two distinct types,

(b) there exists a Hintikka tree over $\{1, \ldots, k\}^{*}$ satisfying $C$, where $k=l(C)$.

Proof. (a) $\Rightarrow$ (b) Suppose that $C^{\mathcal{I}} \neq \emptyset$ in some model $\mathcal{I}$ with the triangle inequality and at least two distinct types. We define a Hintikka tree $K$ satisfying $C$ by induction as follows. First take some $w \in C^{\mathcal{I}}$ and set

$$
K(\epsilon)=\left(\mathcal{T}_{V}^{\mathcal{I}}(w), \leq_{w}, d_{w}\right),
$$

where $\left(\mathcal{T}_{V}^{\mathcal{I}}(w), \leq_{w}, d_{w}\right)$ is a bouquet induced by $w$ and a suitable set $V \subseteq W$ containing $\{\ell\}^{\mathcal{I}}$ for all $\ell$ that occur in $C$.

Suppose now that we have already defined $K(\alpha)$, for some $\alpha \in\{1, \ldots, k\}^{*}$ :

$$
K(\alpha)=\left(\mathcal{T}_{V_{\alpha}}^{\mathcal{I}}\left(w_{\alpha}\right), \leq_{w_{\alpha}}, d_{w_{\alpha}}\right),
$$

where $\left(\mathcal{T}_{V_{\alpha}}^{\mathcal{I}}\left(w_{\alpha}\right), \leq_{w_{\alpha}}, d_{w_{\alpha}}\right)$ is induced by $w_{\alpha}$ and a suitable set $V_{\alpha}$. For each $j \in\{1, \ldots, k\}$, choose some $w_{\alpha j} \in V_{\alpha}$ so that $V_{\alpha}=\left\{w_{\alpha 1}, \ldots, w_{\alpha k}\right\}$, and let

$$
K(\alpha j)=\left(\mathcal{T}_{V_{\alpha j}}^{\mathcal{I}}\left(w_{\alpha j}\right), \leq_{w_{\alpha j}}, d_{w_{\alpha j}}\right)
$$

where $\left(\mathcal{T}_{V_{\alpha j}}^{\mathcal{I}}\left(w_{\alpha j}\right), \leq_{w_{\alpha j}}, d_{w_{\alpha j}}\right)$ is the bouquet induced by $w_{\alpha j}$ and a suitable set $V_{\alpha j}$ which contains some $v$ with $T^{\mathcal{I}}(v)=T^{\mathcal{I}}\left(w_{\alpha}\right)$.

It is easy to see that the resulting $K$ is a Hintikka tree satisfying $C$.

(b) $\Rightarrow$ (a) Let $K$ be a Hintikka tree over $\{1, \ldots, k\}^{*}$ satisfying $C$. First we define a distance space $\left(\Delta_{0}, d_{0}\right)$ with the domain $\{1, \ldots, k\}^{*}$ in the following way.

To begin with, take an arbitrary node $\alpha$ and define $d_{0}$ for $\alpha$ and its successors $\alpha 1, \ldots, \alpha k$. Let $\delta$ and $\varepsilon_{0}, \ldots, \varepsilon_{N}$ be defined by (9). For every $s \leq N$, let

$$
I_{\alpha, s}=\left\{i \mid 1 \leq i \leq k, d_{K(\alpha)}\left(T_{K(\alpha i)}\right)=a_{s}^{-}\right\} .
$$

Then we choose $\varepsilon(\alpha, \alpha i) \in\left(\varepsilon_{s}, 2 \varepsilon_{s}\right)$, for all $i \in I_{\alpha, s}$, in such way that

$$
\varepsilon(\alpha, \alpha i) \geq \varepsilon(\alpha, \alpha j) \quad \text { iff } \quad T_{K(\alpha i)} \leq_{K(\alpha)} T_{K(\alpha j)},
$$

for all $i, j \in I_{\alpha, s}$. Now define

$$
d_{0}(\alpha, \alpha i)=\left\{\begin{array}{lll}
a, & \text { if } d_{K(\alpha)}\left(T_{K(\alpha j)}\right)=a \in \mathrm{P}^{+}(C), \\
a-\varepsilon(\alpha, \alpha j), & \text { if } \quad d_{K(\alpha)}\left(T_{K(\alpha j)}\right)=a^{-} \in \mathrm{Q}(C) \backslash \mathrm{P}^{+}(C) .
\end{array}\right.
$$

Now we can extend $d_{0}$ to the whole set $\Delta_{0}$ as follows. Let $d_{0}(\alpha, \alpha)=0, d_{0}(\alpha i, \alpha)=a_{N}$, and, for every $\alpha j_{1} \cdots j_{n}$,

$$
\begin{aligned}
& d_{0}\left(\alpha, \alpha j_{1} \cdots j_{n}\right)=d_{0}\left(\alpha, \alpha j_{1}\right)+\cdots+d_{0}\left(\alpha j_{1} \cdots j_{n-1}, \alpha j_{1} \cdots j_{n}\right), \\
& d_{0}\left(\alpha j_{1} \cdots j_{n}, \alpha\right)=d_{0}\left(\alpha j_{1} \cdots j_{n}, \alpha j_{1} \cdots j_{n-1}\right)+\cdots+d_{0}\left(\alpha j_{1}, \alpha\right) .
\end{aligned}
$$

Finally, for every two nodes $\alpha j_{1} \cdots j_{n}$ and $\alpha j_{1}^{\prime} \cdots j_{n^{\prime}}^{\prime}$ such that $j_{1} \neq j_{1}^{\prime}$, we let

$$
d_{0}\left(\alpha j_{1} \cdots j_{n}, \alpha j_{1}^{\prime} \cdots j_{n^{\prime}}^{\prime}\right)=d_{0}\left(\alpha j_{1} \cdots j_{n}, \alpha\right)+d_{0}\left(\alpha, \alpha j_{1}^{\prime} \cdots j_{n^{\prime}}^{\prime}\right) .
$$


One can easily see that

$$
T_{K(\alpha i)} \leq_{K(\alpha)} T_{K(\alpha j)} \quad \text { iff } \quad d_{0}(\alpha, \alpha i) \leq d_{0}(\alpha, \alpha j)
$$

for all $\alpha \in\{1, \ldots, k\}^{*}$ and $1 \leq i, j \leq k$.

Finally, we define an interpretation $\mathcal{I}_{0}$ on the space $\left(\Delta_{0}, d_{0}\right)$ by taking, for every concept name $A \in \mathrm{N}_{\mathrm{C}}$ and every object name $\ell \in \mathrm{N}_{\mathrm{O}}$,

$$
\begin{aligned}
A^{\mathcal{I}_{0}} & =\left\{\alpha \in\{1, \ldots, k\}^{*} \mid A \in T_{K(\alpha)}\right\}, \\
\ell^{\mathcal{I}_{0}} & =\left\{\alpha \in\{1, \ldots, k\}^{*} \mid\{\ell\} \in T_{K(\alpha)}\right\} .
\end{aligned}
$$

Observe that this interpretation is not 'real' because object names can be interpreted by non-singleton sets, but let us forget about this for the moment.

Lemma 15. Let $\alpha, \beta \in\{1, \ldots, k\}^{*}$ and $D \in T_{K(\beta)}$, for some $\exists D \in \operatorname{cl}(C)$. Then either $D \in T_{K(\alpha)}$, or $D \in T_{K(\alpha j)}$ and $d_{0}(\alpha, \alpha j) \leq d_{0}(\alpha, \beta)$, for some $j \in\{1, \ldots, k\}$.

Proof. First, using condition (B7), it is not hard to show by induction on the length of $\alpha \in\{1, \ldots, k\}^{*}$ that

$$
\exists C^{\prime} \in T_{K(\alpha)} \text { iff } \exists C^{\prime} \in T_{K(\epsilon)},
$$

for all $\exists C^{\prime} \in \operatorname{cl}(C)$. Therefore, $T_{K(\alpha)}$ contains $\exists D$.

If $D \in T_{K(\alpha)}$ then we are done. Suppose that $D \notin T_{K(\alpha)}$. Then $\alpha \neq \beta$ and we can choose $j \in\{1, \ldots, k\}$ such that $d_{0}(\alpha, \alpha j)$ is minimal with $D \in T_{K(\alpha j)}$.

If $\beta$ is not a successor of $\alpha$ then, by the definition of $d_{0}, d_{0}(\alpha, \alpha i)<a_{N} \leq d_{0}(\alpha, \beta)$, as required. So, let $\beta=\alpha j_{1} \cdots j_{n}$ and proceed by induction on $n$.

$n=1$ : This means that $\beta=\alpha j_{1}$, and we are done again.

$n=2$ : Let $a=d_{0}(\alpha, \alpha j), b=d_{0}\left(\alpha, \alpha j_{1}\right), c=d_{0}\left(\alpha j_{1}, \beta\right)$, and $r, s, t$ be such that

$$
d_{K(\alpha)}\left(T_{K(\alpha j)}\right) \in\left\{a_{r}^{-}, a_{r}\right\}, \quad d_{K(\alpha)}\left(T_{K\left(\alpha j_{1}\right)}\right) \in\left\{a_{s}^{-}, a_{s}\right\}, \quad d_{K\left(\alpha j_{1}\right)}\left(T_{K(\beta)}\right) \in\left\{a_{t}^{-}, a_{t}\right\} .
$$

We need to show $a \leq b+c$. If $a_{r}<a_{s}+a_{t}$ then we have

$$
b+c-a>\left(a_{s}+a_{t}-a_{r}\right)-2 \varepsilon_{s}-2 \varepsilon_{t} \geq \delta-\delta / 2-\delta / 2=0 .
$$

Therefore, we may assume that $a_{s}+a_{t} \leq a_{r}$, in particular $s, t<r$ and $a_{s}+a_{t} \in \mathrm{P}^{+}(C)$. Four cases are possible now: $b<a_{s}, c<a_{t} ; b<a_{s}, c=a_{t} ; b=a_{s}, c<a_{t} ;$ and $b=a_{s}, c=a_{t}$. As all of them are similar, and we only consider the second one as most demonstrative.

Since $D \in T_{K(\beta)}$, we have $\exists^{\leq a_{t}} D \in T_{K\left(\alpha j_{1}\right)}$ by (B6), whence $\exists^{<a_{s}+a_{t}} D \in T_{K(\alpha)}$ by (B8), and $d_{K(\alpha)}\left(T_{K(\alpha j)}\right) \leq\left(a_{s}+a_{t}\right)^{-}$in view of (B5) and by the choice of $j$. Thus, $a_{r}=a_{s}+a_{t}$, $a=a_{r}^{-}$, and

$$
b+c-a>-2 \varepsilon_{s}-2 \varepsilon_{t}+\varepsilon_{r} \geq 0,
$$

since $\varepsilon_{r}, \varepsilon_{s} \leq \varepsilon_{r} / 4$.

$n>2$ : Let $\alpha^{\prime}=\alpha j_{1}$. By the induction hypothesis, there exists $j^{\prime} \in\{1, \ldots, k\}$ such that $D \in T_{K\left(\alpha^{\prime} j^{\prime}\right)}$ and $d_{0}\left(\alpha^{\prime}, \alpha^{\prime} j^{\prime}\right) \leq d_{0}\left(\alpha^{\prime}, \beta\right)$. Again by the induction hypothesis, there exists $j \in\{1, \ldots, k\}$ such that $D \in T_{K(\alpha j)}$ and $d_{0}(\alpha, \alpha j) \leq d_{0}\left(\alpha, \alpha^{\prime} j^{\prime}\right)$. Hence

$$
d_{0}(\alpha, \alpha j) \leq d_{0}\left(\alpha, \alpha^{\prime}\right)+d_{0}\left(\alpha^{\prime}, \alpha^{\prime} j^{\prime}\right) \leq d_{0}\left(\alpha, \alpha^{\prime}\right)+d_{0}\left(\alpha^{\prime}, \beta\right)=d_{0}(\alpha, \beta),
$$

according to the definition of $d_{0}$. 
Lemma 16. For every $D \in \operatorname{cl}(C)$, we have $\alpha \in D^{\mathcal{I}_{0}}$ iff $D \in T_{K(\alpha)}$.

Proof. The proof is by induction on the construction of $D$. The basis of induction and the Boolean cases are trivial.

Case $D=D_{0} \leftleftarrows D_{1}$. Let $\alpha \in\left(D_{0} \leftleftarrows D_{1}\right)^{\mathcal{I}_{0}}$, i.e., $d_{0}\left(\alpha, D_{0}^{\mathcal{I}_{0}}\right)<d_{0}\left(\alpha, D_{1}^{\mathcal{I}_{0}}\right)$. By Lemma 15 ,

$$
d_{0}\left(\alpha, D_{0}^{\mathcal{I}_{0}}\right)=d_{0}\left(\alpha, D_{0}^{\mathcal{I}_{0}} \cap X\right), \quad \text { where } X=\{\alpha\} \cup\{\alpha j \mid 1 \leq j \leq k\} .
$$

Hence, there exists $\beta \in D_{0}^{\mathcal{I}_{0}} \cap X$ such that $d_{0}(\alpha, \beta)<d_{0}\left(\alpha, \beta^{\prime}\right)$ for all $\beta^{\prime} \in D_{1}^{\mathcal{I}_{0}} \cap X$. By the induction hypothesis and (13), this means that $D_{0} \in T_{K(\beta)}$ and $T_{K(\beta)}<_{K(\alpha)} T$, for all $T \in \mathcal{T}_{K(\alpha)}$ with $D_{1} \in T$. Thus $D_{0} \leftleftarrows D_{1} \in T_{K(\alpha)}$ by (B4).

For the converse, suppose $D_{0} \leftleftarrows D_{1} \in T_{K(\alpha)}$, i.e., by (B4), there exists $T \in \mathcal{T}_{K(\alpha)}$ such that $D_{0} \in T$ and $T<_{K(\alpha)} T^{\prime}$ for all $T^{\prime} \in \mathcal{T}_{K(\alpha)}$ with $D_{1} \in T^{\prime}$. Let $T=T_{K\left(\alpha^{\prime}\right)}$ for some $\alpha^{\prime} \in X$. Then $\alpha^{\prime} \in D_{0}^{\mathcal{I}_{0}}$ by the induction hypothesis. If $D_{1}^{\mathcal{I}_{0}}=\emptyset$ then $\alpha \in\left(D_{0} \leftleftarrows D_{1}\right)^{\mathcal{I}_{0}}$ holds trivially. Let $D_{1}^{\mathcal{I}_{0}} \neq \emptyset$. By Lemma 15 , there is $\beta^{\prime} \in D_{1}^{\mathcal{I}_{0}} \cap X$ such that $d_{0}\left(\alpha, D_{1}^{\mathcal{I}_{0}}\right)=d_{0}\left(\alpha, \beta^{\prime}\right)$. By the induction hypothesis, we have $D_{1} \in T_{K\left(\beta^{\prime}\right)}$, and so $T_{K\left(\alpha^{\prime}\right)}<_{K(\alpha)} T_{K\left(\beta^{\prime}\right)}$, whence $d_{0}\left(\alpha, \alpha^{\prime}\right)<d_{0}\left(\alpha, \beta^{\prime}\right)$ by (13). But then $\alpha \in\left(D_{0} \leftleftarrows D_{1}\right)^{\mathcal{I}_{0}}$ by the choice of $\alpha^{\prime}$ and $\beta^{\prime}$.

Case $D=\exists^{<a} D_{0}$. Let $\alpha \in\left(\exists^{<a} D_{0}\right)^{\mathcal{I}_{0}}$, i.e., $d_{0}\left(\alpha, D_{0}^{\mathcal{I}_{0}}\right)<a$. By Lemma 15 , we have (14). Hence there exists $\beta \in D_{0}^{\mathcal{I}_{0}} \cap X$ such that $d_{0}(\alpha, \beta)<a$. By the induction hypothesis and the definition of $d_{0}$, we obtain that $D_{0} \in T_{K(\beta)}$ and $d_{K(\alpha)}\left(T_{K(\beta)}\right) \leq a^{-}$. Thus $\exists^{<a} D_{0} \in T_{K(\alpha)}$ by (B5).

For the converse, let $\exists^{<a} D_{0} \in T_{K(\alpha)}$, i.e., by (B4), $d_{K(\alpha)}(T) \leq a^{-}$and $D_{0} \in T$ for some $T \in \mathcal{T}_{K(\alpha)}$. Let $T=T_{K\left(\alpha^{\prime}\right)}$ for some $\alpha^{\prime} \in X$. Then $\alpha^{\prime} \in D_{0}^{\mathcal{I}_{0}}$ by the induction hypothesis, and $d_{0}\left(\alpha, \alpha^{\prime}\right)<a$ by the definition of $d_{0}$. But then $\alpha \in\left(\exists^{<a} D_{0}\right)^{\mathcal{I}_{0}}$.

Case $D=\exists^{\leq a} D_{0}$ is similar to the previous one.

Thus, it remains to fix the problem with nominals, which can be done as follows. We apply to the pseudo-interpretation $\mathcal{I}_{0}$ the 'filtration' procedure described in the proof of Theorem 4 . Since, by definition, the Hintikka tree $K$ is labelled by a nominal ready set of bouquets and since the filtration results in a model where each type can be realised only once, we then obtain an $\mathcal{S} \mathcal{L}$-model satisfying $C$ where each set of the form $\{\ell\}$ is interpreted by a singleton set.

We are now in a position to prove the ExpTime upper bound by a reduction to the emptiness problem for finite looping tree automata; see [36, 33]. Recall that a finite looping tree automaton $\mathcal{A}$ for infinite $k$-ary trees is a quadruple $\left(\Sigma, Q, \Gamma, Q_{0}\right)$, where

- $\Sigma$ is a (nonempty) finite alphabet,

- $Q$ is a (nonempty) finite set of states of the automaton,

- $\Gamma \subseteq \Sigma \times Q \times Q^{k}$ is a transition relation,

- $Q_{0} \subseteq Q$ is a (nonempty) set of start states of the automaton.

Let $T$ be a $\Sigma$-labelled tree over $\{1, \ldots, k\}^{*}$. A run of $\mathcal{A}$ on $T$ is a function $R:\{1, \ldots, k\}^{*} \rightarrow Q$ such that

- $R(\epsilon) \in Q_{0}$, and

- $(T(\alpha), R(\alpha),(R(\alpha 1), \ldots, R(\alpha k))) \in \Gamma$ for all nodes $\alpha$ of $T$. 
$\mathcal{A}$ accepts $T$ if there exists a run $R$ of $\mathcal{A}$ on $T$. The following emptiness problem for looping automata is decidable in polynomial time [33]: given a looping automaton for $k$-ary trees, decide whether the set of trees it accepts is empty.

To reduce the satisfiability problem for $\mathcal{S} \mathcal{L}$-concepts $C$ in $\mathcal{M}$, we associate with every concept $C$ and every nominal ready set $B$ of bouquets for $C$ a finite looping automaton $\mathcal{A}_{C}^{B}=\left(\Sigma, Q, \Gamma, Q_{0}\right)$ which is defined as follows:

- $\Sigma$ is the set of types occurring in bouquets from $B$,

- $Q=B$,

- $Q_{0}=\left\{\mathfrak{B} \in B \mid C \in T_{\mathfrak{B}}, \mathfrak{B}\right.$ contains a type containing $\ell$, for every $\ell$ in $\left.C\right\}$,

- $\left(T, \mathfrak{B}_{0},\left(\mathfrak{B}_{1}, \ldots, \mathfrak{B}_{k}\right)\right) \in \Gamma$ iff $T_{\mathfrak{B}_{0}}=T, \mathcal{T}_{\mathfrak{B}_{0}} \backslash\left\{T_{\mathfrak{B}_{0}}\right\}=\left\{T_{\mathfrak{B}_{1}}, \ldots, T_{\mathfrak{B}_{k}}\right\}$, and $T_{\mathfrak{B}_{0}} \in \mathcal{T}_{\mathfrak{B}_{i}}$, for $1 \leq i \leq k$.

It follows immediately from Theorem 14 and the given definitions that the runs of $\mathcal{A}_{C}^{B}$ on $\Sigma$-labelled trees are exactly the $B$-labelled Hintikka trees satisfying $C$.

Lemma 17. A concept $C$ is satisfiable in a min-model (with at least two types) iff there exists a nominal ready set $B$ such that $\mathcal{A}_{C}^{B}$ accepts at least one tree.

As there are only exponentially many different nominal ready sets $B$ and as $\mathcal{A}_{C}^{B}$ is only exponential in $l(C)$, the satisfiability problem in min- (and finite) models which satisfy the triangle inequality is decidable in ExpTime.

Symmetric case. The construction of the min-model in the proof of Theorem 14 cannot be directly extended to the symmetric case because we had there $d_{0}(\alpha i, \alpha)=a_{N}>d_{0}(\alpha, \alpha i)$. The straightforward idea of defining $d_{0}(\alpha i, \alpha)=d_{0}(\alpha, \alpha i)$ could lead to the situation where $d_{0}(\alpha, \alpha i) \geq d_{0}(\alpha i, \alpha i j) \geq d_{0}(\alpha i j, \alpha i j k) \geq \ldots$ and infinitely many $\geq$ are actually $>$. The corresponding metric model would not be then a min-model. Our first task is to guarantee that such situations are impossible.

With each Hintikka tree $K$ we associate a certain node-colouring $c$. The set of colours is: inc (for increasing), const (for constant), and dec (for decreasing), and $c$ is a map from $\{1, \ldots, k\}^{*}$ to $\{$ inc, const, dec $\}$ defined by the following rules. The root $\epsilon$ and its immediate successors $1, \ldots, k$ are coloured in the same way, say, $c(\epsilon)=c(1)=\cdots=c(k)=$ inc. And for every $\alpha i j \in\{1, \ldots, k\}^{*}$ we set

$$
c(\alpha i j)= \begin{cases}\text { dec, } & \text { if } T_{K(\alpha i j)}<_{K(\alpha i)} T_{K(\alpha)}, \\ \text { const, } & \text { if } T_{K(\alpha i j)} \simeq_{K(\alpha i)} T_{K(\alpha)}, \\ \text { inc, } & \text { if } T_{K(\alpha)}<_{K(\alpha i)} T_{K(\alpha i j)} .\end{cases}
$$

We call $K$ a min-tree if the following conditions hold:

$$
d_{K(\alpha i)}\left(T_{K(\alpha)}\right) \leq d_{K(\alpha)}\left(T_{K(\alpha i)}\right), \text { for all } \alpha \in\{1, \ldots, k\}^{*} \text { and } 1 \leq i \leq k,
$$

(H5) every branch with infinitely many dec nodes also contains infinitely many inc nodes.

Note first that we can obviously 'prune' Hintikka trees with 'loops' in the following sense: 
Lemma 18. Let $K$ be a Hintikka tree satisfying $C$, and let $\alpha_{0}, \alpha_{1}, \ldots, \alpha_{n}, \alpha_{n+1}$ be a path in $K$ such that $\left(K\left(\alpha_{0}\right), K\left(\alpha_{1}\right)\right)=\left(K\left(\alpha_{n}\right), K\left(\alpha_{n+1}\right)\right)$. For $\beta \in\{1, \ldots, k\}^{*}$, let

$$
K^{\sharp}(\beta)=K\left(\beta^{\sharp}\right), \quad \text { where } \quad \beta^{\sharp}= \begin{cases}\alpha_{n+1} \beta_{1}, & \text { if } \beta=\alpha_{1} \beta_{1} \text { for some } \beta_{1} \neq \epsilon, \\ \beta, & \text { otherwise. }\end{cases}
$$

Then $K^{\sharp}$ is also a Hintikka tree satisfying $C$, and $c^{\sharp}(\beta)=c\left(\beta^{\sharp}\right)$, for all $\beta \in\{1, \ldots, k\}^{*}$, where $c^{\sharp}$ and $c$ are the colourings of $K^{\sharp}$ and $K$, respectively.

Let $K$ be a Hintikka tree and $\bar{\alpha}=\left(\alpha_{1}, \ldots, \alpha_{n}\right)$ be a path in $K$. We say that $\bar{\alpha}$ is a descending path of degree $m$ if $c\left(\alpha_{1}\right), \ldots, c\left(\alpha_{n}\right) \in\{\mathrm{dec}$, const $\}$ and $\bar{\alpha}$ contains exactly $m$ nodes coloured with dec.

Lemma 19. Let $K$ be a min-tree over $\{1, \ldots, k\}^{*}$ satisfying $C$. Let $h=b_{C}^{2}$, where $b_{C}$ is the number of all bouquets for $C$. Then there exists a min-tree $H$ over $\{1, \ldots, k\}^{*}$ satisfying $C$ and such that every descending path in $H$ is of degree $<h$.

Proof. Call a descending path $\alpha_{0}, \ldots, \alpha_{n+1}$ in $K$ special if $n>1, c\left(\alpha_{1}\right)=c\left(\alpha_{n+1}\right)=$ dec, and $\left(K\left(\alpha_{0}\right), K\left(\alpha_{1}\right)\right)=\left(K\left(\alpha_{n}\right), K\left(\alpha_{n+1}\right)\right)$. We say that $\alpha_{0}, \ldots, \alpha_{n+1}$ is maximal if no path of the form $\alpha_{0}, \ldots, \alpha_{r}$ with $r>n+1$ is special in $K$. In view of (H5), every special path in $K$ is contained in a maximal one.

We note also that every descending path $\beta_{1}, \ldots, \beta_{l}$ of degree $\geq h$ gives rise to some special path. Indeed, let $\beta_{0}$ be the immediate predecessor of $\beta_{1}$. Since $K(\alpha) \neq K(\alpha i)$ for all $\alpha i \in$ $\{1, \ldots, k\}^{*}$, the number of possible combinations $\left(K\left(\beta_{m-1}\right), K\left(\beta_{m}\right)\right)$ is less than $h$, i.e., less than the number of $\beta_{m}$ with $c\left(\beta_{m}\right)=$ dec. Therefore $\left(K\left(\beta_{m-1}\right), K\left(\beta_{m}\right)\right)=\left(K\left(\beta_{n-1}\right), K\left(\beta_{n}\right)\right)$ for some $1 \leq m<n \leq l$ with $c\left(\beta_{m}\right)=c\left(\beta_{n}\right)=$ dec, i.e., $\beta_{m-1}, \ldots, \beta_{n}$ is a special path.

Let $\alpha_{0}$ be a node of minimal length in $K$ that starts some special path. Let $\alpha_{0}, \ldots, \alpha_{n+1}$ be a maximal special path. By pruning the tree as described in Lemma 18, we obtain a min-tree $K^{\sharp}$ satisfying $C$ and such that no special path in $K^{\sharp}$ can start with the pair $\alpha_{0}, \alpha_{1}$ or with a node shorter than $\alpha_{0}$. Indeed, suppose that $\alpha_{0}, \ldots, \alpha_{m+1}$ is a special path in $K^{\sharp}$. Then

$$
\alpha_{0}, \alpha_{1}, \ldots, \alpha_{n+1}, \alpha_{2}^{\sharp}, \ldots \alpha_{m}^{\sharp}, \alpha_{m+1}^{\sharp}
$$

is a special path in $K$, contrary to the maximality assumption.

Suppose now that $\beta_{0}, \ldots, \beta_{m+1}$ is a special path in $K^{\sharp}$ such that $\beta_{0}$ is shorter than $\alpha_{0}$. If $\alpha_{1}=\beta_{l}$ for some $l \leq m$ then we arrive to a contradiction in the same way as before. Otherwise, we have $\beta_{l}^{\sharp}=\beta_{l}$ for all $l \leq m+1$. Hence $\beta_{0}, \ldots, \beta_{m+1}$ is a special path in $K$ itself, and $\beta_{0}$ is shorter than $\alpha_{0}$, which is again a contradiction.

Thus we can construct a sequence $K_{0}, K_{1}, K_{2}, \ldots$ of min-trees satisfying $C$ such that, for all $m<n$ in $\mathbb{N}$ and $\alpha \in\{1, \ldots, k\}^{m}$,

$$
K_{0}=K, \quad K_{n}(\alpha)=K_{m}(\alpha), \quad K_{n} \text { contains no special path starting with } \alpha \text {. }
$$

Define a labelled tree $H$ by taking

$$
H(\alpha)=K_{n}(\alpha), \quad \text { whenever } \alpha \in\{1, \ldots, k\}^{n},
$$

for all $n \in \mathbb{N}$. Then $H$ is a min-tree satisfying $C$ and containing no special paths, in particular, no descending paths of degree $\geq h$. 
We are now in a position to prove the following:

Theorem 20. For every concept $C$, the following conditions are equivalent:

(a) $C$ is satisfiable in some metric min-model with at least two distinct types,

(b) there exists a min-tree satisfying $C$ over $\{1, \ldots, k\}^{*}$, where $k=l(C)$.

Proof. (a) $\Rightarrow$ (b) Suppose that $C^{\mathcal{I}} \neq \emptyset$ in some metric model $\mathcal{I}$ with at least two distinct types. Let $K$ be the Hintikka tree $K$ constructed in the same way as in the proof of Theorem 14, (a) $\Rightarrow(\mathrm{b})$. We only need to show that (H4) and (H5) hold for $K$.

(H4) follows from the requirement $T_{K(\alpha)} \in \mathcal{T}_{K(\alpha i)}$ for all $\alpha i \in\{1, \ldots, k\}^{*}$ [see (H3)] and the construction of bouquets.

Now suppose that (H5) does not hold, i.e., there exists a branch $\left(\alpha_{m} \mid m \in \mathbb{N}\right)$ in $K$ such that $c\left(\alpha_{m}\right) \in\{\mathrm{dec}$, const $\}$ for all $m$, and $c\left(\alpha_{m}\right)=\operatorname{dec}$, for infinitely many $m$. As the set $\left\{T^{\mathcal{I}}\left(\alpha_{m}\right) \mid m \in \mathbb{N}\right\}$ is finite and $T_{K\left(\alpha_{m}\right)} \neq T_{K\left(\alpha_{m+1}\right)}$ for every $m$, we must have two distinct types $T, T^{\prime}$ such that the set of pairs

$$
X=\left\{\left(\alpha_{m}, \alpha_{m+1}\right) \mid\left(T_{K\left(\alpha_{m}\right)}, T_{K\left(\alpha_{m+1}\right)}\right)=\left(T, T^{\prime}\right)\right\}
$$

is infinite. For each $m \in \mathbb{N}$, take the point $w_{\alpha_{m}} \in \Delta^{\mathcal{I}}$ which induces the node $\alpha_{m}$. As $\mathcal{I}$ is symmetric and by the construction of bouquets, we have $d\left(w_{\alpha_{m}}, w_{\alpha_{m+1}}\right) \geq d\left(w_{\alpha_{m+1}}, w_{\alpha_{m+2}}\right)$ whenever $c\left(\alpha_{m+2}\right)=$ const, and $d\left(w_{\alpha_{m}}, w_{\alpha_{m+1}}\right)>d\left(w_{\alpha_{m+1}}, w_{\alpha_{m+2}}\right)$ whenever $c\left(\alpha_{m+2}\right)=$ dec. Now let $Z \subseteq \Delta^{\mathcal{I}}$ and $Z^{\prime} \subseteq \Delta^{\mathcal{I}}$ be defined by

$$
Z=\left\{w_{\alpha_{m}} \mid\left(\alpha_{m}, \alpha_{m+1}\right) \in X\right\} \quad \text { and } \quad Z^{\prime}=\left\{w_{\alpha_{m+1}} \mid\left(\alpha_{m}, \alpha_{m+1}\right) \in X\right\} .
$$

As $\mathcal{I}$ is a min-model, there are points $u \in Z$ and $u^{\prime} \in Z^{\prime}$ such that $d^{\mathcal{I}}\left(u, u^{\prime}\right)=d^{\mathcal{I}}\left(Z, Z^{\prime}\right)$. By the definition of bouquets induced by points of $\mathcal{I}$, we may assume that $\left(u, u^{\prime}\right)$ gives rise to a $\left(\alpha_{r}, \alpha_{r+1}\right) \in X$. Now take some $\left(\alpha_{s}, \alpha_{s+1}\right) \in X$ such that $s>r$ and a dec node occurs between $\alpha_{r}$ and $\alpha_{s}$. Let $v$ and $v^{\prime}$ be the points from $\mathcal{I}$ inducing $\alpha_{s}$ and $\alpha_{s+1}$. Since all $\alpha_{m}, m \in \mathbb{N}$, are dec or const nodes we come to the conclusion that $d^{\mathcal{I}}\left(v, v^{\prime}\right)<d^{\mathcal{I}}\left(u, u^{\prime}\right)=d^{\mathcal{I}}\left(Z, Z^{\prime}\right)$, which is a contradiction.

(b) $\Rightarrow$ (a) Let $K$ be a min-tree satisfying $C$. In view of Lemma 19, we can assume that every descending path in $K$ is of degree $<h=b_{C}^{2}$.

Define a metric space $\left(\Delta_{1}, d_{1}\right)$ over $\{1, \ldots, k\}^{*}$ in the following way. First, we introduce $d_{1}$ for every node and its successors by induction. Let $\delta$ and $\varepsilon_{0}, \ldots, \varepsilon_{N}$ be defined by (9). For every $s \leq N$, let $E_{s}$ be an arbitrary fixed subset of $\left(\varepsilon_{s}, 2 \varepsilon_{s}\right)$ of cardinality $h \cdot k$, and let $F_{s}$ be a countable subset of $\left(\varepsilon_{s}, \min E_{s}\right)$ with the order type of the negative integers.

For every $\alpha \in\{1, \ldots, k\}^{*}$, set

$$
I_{\alpha, s}=\left\{i \mid 1 \leq i \leq k, d_{K(\alpha)}\left(T_{K(\alpha i)}\right)=a_{s}^{-}\right\} .
$$

For every $s \leq N$, choose $\varepsilon(\epsilon, i) \in F_{s}$, for $i \in I_{\epsilon, s}$, in such a way that

$$
\varepsilon(\epsilon, i) \geq \varepsilon(\epsilon, j) \quad \text { iff } \quad T_{K(i)} \leq_{K(\epsilon)} T_{K(j)},
$$

for all $i, j \in I_{\epsilon, s}$. Then we define

$$
d_{1}(\epsilon, i)=\left\{\begin{array}{lll}
a, & \text { if } \quad d_{K(\epsilon)}\left(T_{K(j)}\right)=a \in \mathrm{P}^{+}(C), \\
a-\varepsilon(\epsilon, j), & \text { if } \quad d_{K(\epsilon)}\left(T_{K(j)}\right)=a^{-} \in \mathrm{Q}(C) \backslash \mathrm{P}^{+}(C) .
\end{array}\right.
$$

Now suppose that $d_{1}\left(\alpha^{\prime}, \alpha\right)$ is already defined, where $\alpha^{\prime}$ is the immediate predecessor of $\alpha$. For all $s \leq N$, choose $\varepsilon(\alpha, \alpha i) \in E_{s} \cup F_{s}$, for $i \in I_{\alpha, s}$, satisfying the following conditions: 
(D1) if $c(\alpha i)=$ const and $d_{1}\left(\alpha^{\prime}, \alpha\right) \geq a_{s}$, then $\varepsilon(\alpha, \alpha i) \in F_{s}$,

(D2) if $c(\alpha i)=$ const and $d_{1}\left(\alpha^{\prime}, \alpha\right)<a_{s}$, then $\varepsilon(\alpha, \alpha i)=\varepsilon\left(\alpha^{\prime}, \alpha\right)=a_{s}-d_{1}\left(\alpha^{\prime}, \alpha\right)$,

(D3) if $c(\alpha i)=$ inc, then $\varepsilon(\alpha, \alpha i) \in F_{s}$,

(D4) if $c(\alpha i)=\mathrm{dec}$, then

$$
\varepsilon(\alpha, \alpha i)=\min \left\{x \in E_{s} \mid x>\varepsilon(\alpha, \alpha j) \text {, for all } j \in I_{\alpha, s} \text { with } T_{K(\alpha i)}<_{K(\alpha)} T_{K(\alpha j)}\right\},
$$

(D5) $\varepsilon(\alpha, \alpha i) \geq \varepsilon(\alpha, \alpha j)$ iff $T_{K(\alpha i)} \leq_{K(\alpha)} T_{K(\alpha j)}$, for all $i, j \in I_{\alpha, s}$.

The next lemma shows that the sets $E_{s}$ are large enough to satisfy these conditions.

Lemma 21. Let $\varepsilon(\alpha, \alpha i) \in E_{s}$, for some $\alpha i \in\{1, \ldots, k\}$ and $s \leq N$. Then $\alpha i$ is the end of some descending path of positive degree. Let $m$ be degree of the longest such path, and $E(\alpha i)$ denote the set $\left\{x \in E_{s} \mid x \leq \varepsilon(\alpha, \alpha i)\right\}$. Then $|E(\alpha i)| \leq m \cdot k$.

Proof. Note first that $\alpha$ cannot be empty, since $\varepsilon(\alpha, \alpha i) \notin F_{s}$. Let $\alpha^{\prime}$ be the immediate predecessor of $\alpha$.

Claim 1. Let $\alpha_{1}, \ldots, \alpha_{n+1}$ be a path such that $\alpha_{n+1}=\alpha i$ and $c\left(\alpha_{1}\right) \neq$ const $=c\left(\alpha_{m}\right)$, for all $2 \leq m \leq n+1$. Then $c\left(\alpha_{1}\right)=\operatorname{dec}$ and $\varepsilon\left(\alpha_{m}, \alpha_{m+1}\right)=\varepsilon(\alpha, \alpha i)$, for all $m<n$.

The proof is by induction on $n$.

Case $n=0$ : then $c(\alpha i) \neq$ const. But $c(\alpha i)=$ inc implies $\varepsilon(\alpha, \alpha i) \in F_{s}$, contrary to $\varepsilon(\alpha, \alpha i) \in E_{s}$. Hence $c(\alpha i)=\mathrm{dec}$, as required.

Case $n>0$ : then $c(\alpha i)=$ const, i.e., $T_{K(\alpha i)} \simeq_{K(\alpha)} T_{K\left(\alpha^{\prime}\right)}$. By applying (B2) and (H4) we obtain $a_{s}^{-}=d_{K(\alpha)}\left(T_{K(\alpha i)}\right)=d_{K(\alpha)}\left(T_{K\left(\alpha^{\prime}\right)}\right) \leq d_{K\left(\alpha^{\prime}\right)}\left(T_{K(\alpha)}\right)$. So we have either $d_{1}\left(\alpha^{\prime}, \alpha\right) \geq a_{s}$, or $d_{1}\left(\alpha^{\prime}, \alpha\right)=a_{s}-\varepsilon\left(\alpha^{\prime}, \alpha\right)$. In the former case we obtain $\varepsilon(\alpha, \alpha i) \in F_{s}$ by (D1), contrary to $\varepsilon(\alpha, \alpha i) \in E_{s}$. Therefore $\varepsilon(\alpha, \alpha i)=\varepsilon\left(\alpha^{\prime}, \alpha\right)$ by (D2), i.e., $\varepsilon\left(\alpha^{\prime}, \alpha\right) \in E_{s}$, and we are done by the induction hypothesis (note that $\alpha^{\prime}=\alpha_{n-1}$ ).

Claim 2. Let $E^{\prime}(\alpha i)=\left\{x \in E_{s} \mid a_{s}-d_{1}\left(\alpha^{\prime}, \alpha\right)<x \leq \varepsilon(\alpha, \alpha i)\right\}$. Then $\left|E^{\prime}(\alpha i)\right| \leq k$.

In view of Claim 1 , we have $c(\alpha i) \in\{$ dec, const $\}$. Suppose that $c(\alpha i)=$ const. Then, again by Claim 1 , we obtain that $a_{s}-d_{1}\left(\alpha, \alpha^{\prime}\right)=\varepsilon\left(\alpha^{\prime}, \alpha\right)=\varepsilon(\alpha, \alpha i)$, i.e., $\left|E^{\prime}(\alpha i)\right|=0$.

We assume therefore that $c(\alpha i)=$ dec. Then $T_{K\left(\alpha^{\prime}\right)}<_{K(\alpha)} T_{K(\alpha i)}$ and by applying (B2) and (H4) we obtain $a_{s}^{-}=d_{K(\alpha)}\left(T_{K(\alpha i)}\right) \leq d_{K(\alpha)}\left(T_{K\left(\alpha^{\prime}\right)}\right) \leq d_{K\left(\alpha^{\prime}\right)}\left(T_{K(\alpha)}\right)$. So we have either $d_{1}\left(\alpha^{\prime}, \alpha\right) \geq a_{s}$ or $d_{1}\left(\alpha^{\prime}, \alpha\right)=a_{s}-\varepsilon\left(\alpha^{\prime}, \alpha\right)$.

Consider first the case $d_{1}\left(\alpha^{\prime}, \alpha\right) \geq a_{s}$. Then, for all $j \in I_{\alpha, s}$ such that $c(\alpha j) \in\{$ const, inc $\}$, we obtain $\varepsilon(\alpha, \alpha j) \in F_{s}$ by $(D 1)$ or $(D 3)$. Let

$$
\left\{j \in I_{\alpha, s} \mid c(\alpha j)=\mathrm{dec}\right\}=\left\{j_{1}, \ldots, j_{l}\right\}, \quad \text { where } T_{K\left(\alpha j_{r}\right)} \leq_{K(\alpha)} T_{K\left(\alpha j_{s}\right)} \text { for } r \leq s .
$$

Then, in view of (D4), we obtain

$$
\begin{aligned}
\varepsilon\left(\alpha, \alpha j_{1}\right) & =\min E_{s}, \\
\varepsilon\left(\alpha, \alpha j_{r+1}\right) & =\varepsilon\left(\alpha, \alpha j_{r}\right) \text { or } \min \left\{x \in E_{s} \mid x>\varepsilon\left(\alpha, \alpha j_{r}\right)\right\}, \text { for all } r<l .
\end{aligned}
$$

Therefore, $\left|E^{\prime}(\alpha i)\right| \leq\left|I_{\alpha, s}\right| \leq k$. 
Suppose now that $d_{1}\left(\alpha^{\prime}, \alpha\right)=a_{s}-\varepsilon\left(\alpha^{\prime}, \alpha\right)$. Then, for all $j \in I_{\alpha, s}$ with $c(\alpha j) \in\{$ const, inc $\}$, we have $T_{K(\alpha i)} \leq_{K(\alpha)} T_{K\left(\alpha^{\prime}\right)}$, and so $\varepsilon\left(\alpha^{\prime}, \alpha\right) \geq \varepsilon(\alpha, \alpha j)$ by (D5). Consider again (15). Then, in view of (D4), we obtain

$$
\begin{aligned}
\varepsilon\left(\alpha, \alpha j_{1}\right) & =\min \left\{x \in E_{s} \mid x>\varepsilon\left(\alpha^{\prime}, \alpha\right)\right\}, \\
\varepsilon\left(\alpha, \alpha j_{r+1}\right) & =\varepsilon\left(\alpha, \alpha j_{r}\right) \text { or } \min \left\{x \in E_{s} \mid x>\varepsilon\left(\alpha, \alpha j_{r}\right)\right\}, \text { for all } r<l .
\end{aligned}
$$

Therefore, $\left|E^{\prime}(\alpha i)\right| \leq\left|I_{\alpha, s}\right| \leq k$.

Let us now return to the proof of Lemma 21. In view of Claim 1, we can consider the longest descending path $\alpha_{0}, \ldots, \alpha_{n+1}$ with $\alpha_{n+1}=\alpha i$. We prove $|E(\alpha i)| \leq m \cdot k$ by induction on $n$.

Case $n=0$ : then $c(\alpha)=$ inc and, in view of Claim $1, c(\alpha i)=$ dec, i.e., $m=1$. As before, we have either $d_{1}\left(\alpha^{\prime}, \alpha\right) \geq a_{s}$ or $d_{1}\left(\alpha^{\prime}, \alpha\right)=a_{s}-\varepsilon\left(\alpha^{\prime}, \alpha\right)$. And since $c(\alpha)=$ inc, we have $\varepsilon\left(\alpha^{\prime}, \alpha\right) \in F_{s}$ in the latter case by (D5). Therefore, in any case, $a_{s}-d_{1}\left(\alpha^{\prime}, \alpha\right)<x$ for all $x \in E_{s}$. We obtain $|E(\alpha i)| \leq k \leq m \cdot k$ by Claim 2 .

Case $n>0$ : then $c(\alpha i) \in\{\mathrm{dec}$, const $\}$ and $m \geq 1$ by Claim 1 . Suppose first that $c(\alpha i)=$ const. Then, in view of Claim $1, E(\alpha i)=E(\alpha)$ and the degree of the longest descending path which ends at $\alpha$ is $m$ as well. We obtain $|E(\alpha i)| \leq m \cdot k$ by the induction hypothesis.

Suppose now that $c(\alpha i)=$ dec. Then, as before, we have either $d_{1}\left(\alpha^{\prime}, \alpha\right) \geq a_{s}$ or $d_{1}\left(\alpha^{\prime}, \alpha\right)=a_{s}-\varepsilon\left(\alpha^{\prime}, \alpha\right)$. If $d_{1}\left(\alpha^{\prime}, \alpha\right) \geq a_{s}$ or $\varepsilon\left(\alpha^{\prime}, \alpha\right) \in F_{s}$, then $E(\alpha i)=E^{\prime}(\alpha i)$, and so $|E(\alpha i)| \leq k(\leq m \cdot k)$ by Claim 2. Assume therefore that $d_{1}\left(\alpha^{\prime}, \alpha\right)=a_{s}-\varepsilon\left(\alpha^{\prime}, \alpha\right)$, where $\varepsilon\left(\alpha^{\prime}, \alpha\right) \in E_{s}$. Then the longest descending path with the end $\alpha$ is of degree $m-1$. Hence $|E(\alpha)| \leq(m-1) k$ by the induction hypothesis, and $\left|E^{\prime}(\alpha i)\right| \leq k$ by Claim 2 . Finally, we obtain $|E(\alpha i)|=\left|E(\alpha) \cup E^{\prime}(\alpha i)\right| \leq m \cdot k$.

Thus, we can set

$$
d_{1}(\alpha, \alpha i)=\left\{\begin{array}{lll}
a, & \text { if } d_{K(\alpha)}\left(T_{K(\alpha j)}\right)=a \in \mathrm{P}^{+}(\Sigma), \\
a-\varepsilon(\alpha, \alpha j), & \text { if } \quad d_{K(\alpha)}\left(T_{K(\alpha j)}\right)=a^{-} \in \mathrm{Q}(C) \backslash \mathrm{P}^{+}(\Sigma) .
\end{array}\right.
$$

It is not hard to see that

$$
T_{K(\alpha i)} \leq_{K(\alpha)} T_{K(\alpha j)} \quad \text { iff } \quad d_{1}(\alpha, \alpha i) \leq d_{1}(\alpha, \alpha j)
$$

for all $\alpha \in\{1, \ldots, k\}^{*}$ and $1 \leq i, j \leq k$.

We extend $d_{1}$ to the whole tree $K$ as follows. Let $d_{1}(\alpha, \alpha)=0$ and, for every node $\alpha j_{1} \cdots j_{n}$,

$$
d_{1}\left(\alpha, \alpha j_{1} \cdots j_{n}\right)=d_{1}\left(\alpha, \alpha j_{1}\right)+\cdots+d_{1}\left(\alpha j_{1} \cdots j_{n-1}, \alpha j_{1} \cdots j_{n}\right) .
$$

Finally, for every two nodes $\alpha j_{1} \cdots j_{n}$ and $\alpha j_{1}^{\prime} \cdots j_{n^{\prime}}^{\prime}$ such that $j_{1} \neq j_{1}^{\prime}$ we let

$$
d_{1}\left(\alpha j_{1} \cdots j_{n}, \alpha j_{1}^{\prime} \cdots j_{n^{\prime}}^{\prime}\right)=d_{1}\left(\alpha, \alpha j_{1} \cdots j_{n}\right)+d_{1}\left(\alpha, \alpha j_{1}^{\prime} \cdots j_{n^{\prime}}^{\prime}\right) .
$$

All the remaining distances are defined by symmetry.

Now we can we define an interpretation $\mathcal{I}_{1}$ on the space $\left(\Delta_{1}, d_{1}\right)$ by taking, for every concept name $A \in \mathrm{N}_{\mathrm{C}}$ and every object name $\ell \in \mathrm{N}_{\mathrm{O}}$,

$$
\begin{aligned}
A^{\mathcal{I}_{1}} & =\left\{\alpha \in\{1, \ldots, k\}^{*} \mid A \in T_{K(\alpha)}\right\}, \\
\ell^{\mathcal{I}_{1}} & =\left\{\alpha \in\{1, \ldots, k\}^{*} \mid\{\ell\} \in T_{K(\alpha)}\right\} .
\end{aligned}
$$


Observe that, as before, this interpretation is not 'real' because object names can be interpreted by non-singleton sets, but let us forget about this for the moment.

Lemma 22. Let $\alpha, \beta \in\{1, \ldots, k\}^{*}$ and $D \in T_{K(\beta)}$, for some $\exists D \in \operatorname{cl}(C)$. Then either $D \in T_{K(\alpha)}$, or $D \in T_{K(\alpha j)}$ and $d_{1}(\alpha, \alpha j) \leq d_{1}(\alpha, \beta)$, for some $j \in\{1, \ldots, k\}$.

Proof. First, using condition (B7), it is not hard to show by induction on the length of $\alpha \in\{1, \ldots, k\}^{*}$ that

$$
\exists C^{\prime} \in T_{K(\alpha)} \text { iff } \quad \exists C^{\prime} \in T_{K(\epsilon)},
$$

for all $\exists C^{\prime} \in \operatorname{cl}(C)$. Therefore $T_{K(\alpha)}$ contains $\exists D$.

If $D \in T_{K(\alpha)}$ then we are done. So suppose that $D \notin T_{K(\alpha)}$. Then $\alpha \neq \beta$ and there is $j \in\{1, \ldots, k\}$ such that $D \in T_{K(\alpha j)}$.

Let $\alpha=\gamma i_{1} \ldots i_{m}$ and $\beta=\gamma j_{1} \ldots j_{n}$. We proceed by induction on $m+n$.

$m=0, n=1$ : this means that $\beta=\alpha j_{1}$, and so we are done again.

$m=1, n=0$ : this means that $\alpha=\beta i_{1}$, and so $T_{K(\beta)} \in \mathcal{T}_{K(\alpha)}$ by (H3). Take $j \in\{1, \ldots, k\}$ such that $T_{K(\alpha j)}=T_{K(\beta)}$. Then $c(\alpha j)=$ const, and hence $d_{1}(\alpha, \alpha j) \leq d_{1}(\alpha, \beta)$ by (D1) or (D2), as required.

$m=1, n=1$ : choose $j \in\{1, \ldots, k\}$ such that $d_{1}(\alpha, \alpha j)$ is minimal with $D \in T_{K(\alpha j)}$. Let $a=d_{0}(\alpha, \alpha j), b=d_{0}(\gamma, \alpha), c=d_{0}(\gamma, \beta)$, and $r, s, t$ be such that

$$
d_{K(\alpha)}\left(T_{K(\alpha j)}\right) \in\left\{a_{r}^{-}, a_{r}\right\}, \quad d_{K(\gamma)}\left(T_{K(\alpha)}\right) \in\left\{a_{s}^{-}, a_{s}\right\}, \quad d_{K(\gamma)}\left(T_{K(\beta)}\right) \in\left\{a_{t}^{-}, a_{t}\right\} .
$$

We need to show that $a \leq b+c$. If $a_{r}<a_{s}+a_{t}$ then we have

$$
b+c-a>\left(a_{s}+a_{t}-a_{r}\right)-2 \varepsilon_{s}-2 \varepsilon_{t} \geq \delta-\delta / 2-\delta / 2=0 .
$$

Therefore, we may assume that $a_{s}+a_{t} \leq a_{r}$, in particular $s, t<r$ and $a_{s}+a_{t} \in \mathrm{P}^{+}(C)$. Four cases are possible now: $b<a_{s}, c<a_{t} ; \quad b<a_{s}, c=a_{t} ; \quad b=a_{s}, c<a_{t} ;$ and $b=a_{s}$, $c=a_{t}$. As all of them are similar, and we only consider the second one, which is the most demonstrative.

Note first that $d_{K(\alpha)}\left(T_{K(\gamma)}\right) \leq a_{s}^{-}$by (H4). Now, since $D \in T_{K(\beta)}$, we have $\exists \leq a_{t} D \in$ $T_{K(\gamma)}$ by (B6), whence $\exists<a_{s}+a_{t} D \in T_{K(\alpha)}$ by (B8) and the observation above. Therefore, $d_{K(\alpha)}\left(T_{K(\alpha j)}\right) \leq\left(a_{s}+a_{t}\right)^{-}$by (B5) and the choice of $j$. Thus, $a_{r}=a_{s}+a_{t}, a=a_{r}^{-}$, and

$$
b+c-a>-2 \varepsilon_{s}-2 \varepsilon_{t}+\varepsilon_{r} \geq 0
$$

since $\varepsilon_{r}, \varepsilon_{s} \leq \varepsilon_{r} / 4$.

The case $m=2, n=0$ is considered similarly to the previous one, while the case $m=0$, $n=2$ remains the same as in the non-symmetric case.

$m>2$ : Let $\alpha^{\prime}=\gamma i_{1} \ldots i_{m-1}$. By the induction hypothesis, there exists $j^{\prime} \in\{1, \ldots, k\}$ such that $D \in T_{K\left(\alpha^{\prime} j^{\prime}\right)}$ and $d_{1}\left(\alpha^{\prime}, \alpha^{\prime} j^{\prime}\right) \leq d_{1}\left(\alpha^{\prime}, \beta\right)$. Again by the induction hypothesis, there exists $j \in\{1, \ldots, k\}$ such that $D \in T_{K(\alpha j)}$ and $d_{1}(\alpha, \alpha j) \leq d_{1}\left(\alpha, \alpha^{\prime} j^{\prime}\right)$. Hence

$$
d_{1}(\alpha, \alpha j) \leq d_{1}\left(\alpha, \alpha^{\prime}\right)+d_{1}\left(\alpha^{\prime}, \alpha^{\prime} j^{\prime}\right) \leq d_{1}\left(\alpha, \alpha^{\prime}\right)+d_{1}\left(\alpha^{\prime}, \beta\right)=d_{1}(\alpha, \beta),
$$

by the definition of $d_{1}$.

The case $n>2$ is similar. 
Lemma 23. For every $D \in \operatorname{cl}(C)$, we have $\alpha \in D^{\mathcal{I}_{1}}$ iff $D \in T_{K(\alpha)}$

Proof. The proof is similar to the proof of Lemma 16.

Thus, it remains to fix the problem with nominals, which can be done as follows. We apply to the pseudo-interpretation $\mathcal{I}_{1}$ the 'filtration' procedure described in the proof of Theorem 4. Since, by definition, the min-tree $K$ is labelled by a nominal ready set of bouquets and since the filtration results in a model where each type can be realised only once, we then obtain an $\mathcal{S L}$-model satisfying $C$ where each set of the form $\{\ell\}$ is interpreted by a singleton set.

A single complemented pair automaton $\mathcal{A}$ on infinite $k$-ary trees is a tuple $\left(\Sigma, Q, \Gamma, Q_{0}, F\right)$, where

- $\left(\Sigma, Q, \Gamma, Q_{0}\right)$ is a looping tree automaton as defined above,

- $F$ is a pair of disjoint sets of states from $Q$; it will be convenient for us to assume that $F=($ dec, inc) and dec, inc $\subseteq Q$.

$\mathcal{A}$ accepts a $\Sigma$-labelled tree $T$ over $\{1, \ldots, k\}^{*}$ iff there exists a run $R$ of $\mathcal{A}$ on $T$ such that, for every path $i_{0} i_{1} \ldots$ in $T$, if $R\left(i_{0} i_{1} \ldots i_{j}\right) \in$ dec for infinitely many $j$, then $R\left(i_{0} i_{1} \ldots i_{j}\right) \in$ inc for infinitely many $j$ as well.

As was shown in [8], the emptiness problem for single complemented pair automata is decidable in polynomial time. We show now how to reduce the satisfiability problem for $\mathcal{S} \mathcal{L}$-concepts in symmetric models to the emptiness problem for these automata.

A coloured $C$-bouquet is a pair $(\mathfrak{B}, c)$ where $\mathfrak{B}=\left(\mathcal{T}_{\mathfrak{B}}, \leq_{\mathfrak{B}}, d_{\mathfrak{B}}\right)$ is a bouquet for $C$ and $c$ is a function from $\mathcal{T}_{\mathfrak{B}}$ to $\{\mathrm{dec}$, inc, const $\}$.

With every concept $C$ and every nominal ready set $B$ of coloured $C$-bouquets we associate a single complemented pair automaton $\mathcal{A}_{C}^{B}=\left(\Sigma, Q, \Delta, Q_{0}, F\right)$ by taking

- $\Sigma$ to be the set of types occurring in coloured bouquets of $B$,

- $Q=B$,

- $Q_{0}=\left\{(\mathfrak{B}, c) \in B \mid C \in T_{\mathfrak{B}}, \mathfrak{B}\right.$ contains a type with $\ell$ for every $\ell$ in $\left.\tau\right\}$,

- $\operatorname{dec}=\left\{(\mathfrak{B}, c) \in B \mid c\left(T_{\mathfrak{B}}\right)=\operatorname{dec}\right\}$,

- inc $=\left\{(\mathfrak{B}, c) \in B \mid c\left(T_{\mathfrak{B}}\right)=\right.$ inc $\}$,

- $\left(T,\left(\mathfrak{B}_{0}, c_{0}\right),\left(\mathfrak{B}_{1}, c_{1}\right), \ldots,\left(\mathfrak{B}_{k}, c_{k}\right)\right) \in \Gamma \quad$ iff $\quad T_{\mathfrak{B}_{0}}=T, \mathcal{T}_{\mathfrak{B}_{0}} \backslash\left\{T_{\mathfrak{B}_{0}}\right\}=\left\{T_{\mathfrak{B}_{1}}, \ldots, T_{\mathfrak{B}_{k}}\right\}$, $T_{\mathfrak{B}_{0}} \in \mathcal{T}_{\mathfrak{B}_{i}}, c_{i}\left(T_{\mathfrak{B}_{i}}\right)=c_{0}\left(T_{\mathfrak{B}_{i}}\right)$, and $d_{\mathfrak{B}_{i}}\left(T_{\mathfrak{B}_{0}}\right) \leq d_{\mathfrak{B}_{0}}\left(T_{\mathfrak{B}_{i}}\right)$ for $1 \leq i \leq k$, and for all $T^{\prime} \in \mathcal{T}_{\mathfrak{B}_{i}} \backslash\left\{T_{\mathfrak{B}_{i}}\right\}$

$$
\begin{aligned}
& -c_{i}\left(T^{\prime}\right)=\text { inc iff } \quad T<_{\mathfrak{B}_{i}} T^{\prime}, \\
& -c_{i}\left(T^{\prime}\right)=\text { const iff } T^{\prime} \sim_{\mathfrak{B}_{i}} T, \\
& -c_{i}\left(T^{\prime}\right)=\text { dec iff } T^{\prime}<_{\mathfrak{B}_{i}} T .
\end{aligned}
$$

It follows immediately from Theorem 20 and the given definitions that the runs of $\mathcal{A}_{C}^{B}$ on $\Sigma$-labelled trees are exactly the $B$-labelled Hintikka-trees satisfying $C$.

Lemma 24. A concept $C$ is satisfiable in a symmetric min-model (with at least two distinct types) iff there exists a nominal ready set $B$ of coloured $C$-bouquets such that $\mathcal{A}_{C}^{B}$ accepts at least one tree.

As there are only exponentially many different nominal ready sets $B$ of coloured $C$ bouquets and as $\mathcal{A}_{C}^{B}$ is only exponential in $l(C)$, the satisfiability problem in symmetric min-models is decidable in ExpTime. 


\subsection{Proof of Theorem 3 (ii)}

As in the proof of Theorem 3 (iii), we show this result for types over an $\mathcal{S} \mathcal{L}$-concept $C$ rather than types over a knowledge base $\Sigma$. Suppose that a set $\mathcal{T}$ of types over a concept $C$ is given. We first consider the problem of checking realisability in a min-space satisfying the triangle inequality (but not necessarily symmetric).

Denote by $B$ the set of bouquets for $C$ with types from $\mathcal{T}$. For every $T_{0} \in \mathcal{T}$ construct a finite looping automaton $\mathcal{A}_{T_{0}}=\left(\Sigma, Q, \Gamma, Q_{0}\right)$ as follows:

- $\Sigma$ is the set $\mathcal{T}$,

- $Q=B$,

- $Q_{0}=\left\{\mathfrak{B} \in B \mid T_{0} \in T_{\mathfrak{B}}\right\}$,

- $\left(T, \mathfrak{B}_{0},\left(\mathfrak{B}_{1}, \ldots, \mathfrak{B}_{k}\right)\right) \in \Gamma$ iff $T_{\mathfrak{B}_{0}}=T, \mathcal{T}_{\mathfrak{B}_{0}} \backslash\left\{T_{\mathfrak{B}_{0}}\right\}=\left\{T_{\mathfrak{B}_{1}}, \ldots, T_{\mathfrak{B}_{k}}\right\}$, and $T_{\mathfrak{B}_{0}} \in \mathcal{T}_{\mathfrak{B}_{i}}$, for $1 \leq i \leq k$.

Now the decision procedure runs as follows:

- Check whether $\mathcal{T}$ is nominal ready in the sense that for each $\ell$ in $C$ there exists only one $T \in \mathcal{T}$ such that $\{\ell\} \in T$. If this is not case, then $\mathcal{T}$ is not realisable. Otherwise we go to the next step.

- Check whether for each $\exists D \in \operatorname{cl}(C)$, if there exists $T \in \mathcal{T}$ such that $\exists D \in T$, then $\exists D \in T^{\prime}$ for all $T^{\prime} \in \mathcal{T}$. If this is not the case, then $\mathcal{T}$ is not realisable. Otherwise we go to the next step.

- Check, for each $T \in \mathcal{T}$, whether (i) $T$ is satisfied in a min-model with one point or (ii) $\mathcal{A}_{T}$ accepts at least one tree. If (i) or (ii) hold for all $T \in \mathcal{T}$, then $\mathcal{T}$ is realisable. Otherwise it is not realisable.

It follows immediately from our previous results that if this procedure states that $\mathcal{T}$ is not realisable, then $\mathcal{T}$ is not realisable indeed. Moreover, the procedure clearly terminates after exponentially many steps. Now suppose that the procedure claims that $\mathcal{T}$ is realisable. By the definition of $\mathcal{A}_{T}$, for each $T \in \mathcal{T}$ there exists a min-model $\mathcal{I}_{T}$ which realises a subset of $\mathcal{T}$ containing $T$. In view of the finite model property we can assume all these models to be finite.

We first construct a 'disjoint union' $\mathcal{I}$ of the models $\mathcal{I}_{T}, T \in \mathcal{T}$, as follows. Take

$$
d=\max \left\{d^{\mathcal{I}_{T}}(u, v) \mid u, v \in \Delta^{\mathcal{I}_{T}}, T \in \mathcal{T}\right\},
$$

and then define

$$
\begin{aligned}
& \Delta^{\mathcal{I}}=\bigcup\left\{\Delta^{\mathcal{I}_{T}} \times\{T\} \mid T \in \mathcal{T}\right\}, \\
& d^{\mathcal{I}}\left((u, T),\left(v, T^{\prime}\right)\right)= \begin{cases}d^{\mathcal{I}_{T}}(u, v), & \text { if } T=T^{\prime}, \\
d+1, & \text { if } T \neq T^{\prime},\end{cases} \\
& \ell^{\mathcal{I}}=\left\{\left(\ell^{\mathcal{I}_{T}}, T\right) \mid T \in \mathcal{T}\right\}, \quad \text { for every object name } \ell, \\
& A^{\mathcal{I}}=\bigcup\left\{A^{\mathcal{I}_{T}} \times\{T\} \mid T \in \mathcal{T}\right\}, \quad \text { for every concept name } A .
\end{aligned}
$$

It is not hard to check that $\mathcal{I}$ is a min-model with (tr) which satisfies exactly the types from $\mathcal{T}$. However, the object names may be interpreted by non-singleton sets in $\mathcal{I}$. Therefore we apply to $\mathcal{I}$ the 'filtration' procedure above to construct the required model $\overline{\mathcal{I}}$. 
Let us now consider the problem of realisability in metric min-spaces. Denote by $B^{\prime}$ the set of coloured bouquets with types from $\mathcal{T}$. For every $T_{0} \in \mathcal{T}$ construct a single complemented pair automaton $\mathcal{A}_{T_{0}}^{\prime}=\left(\Sigma, Q, \Gamma, Q_{0}, F\right)$ as follows:

- $\Sigma$ is the set $\mathcal{T}$,

- $Q=B^{\prime}$

- $Q_{0}=\left\{(\mathfrak{B}, c) \in B^{\prime} \mid T_{0} \in T_{\mathfrak{B}}\right\}$,

- $\operatorname{dec}=\left\{(\mathfrak{B}, c) \in B \mid c\left(T_{\mathfrak{B}}\right)=\operatorname{dec}\right\}$,

- inc $=\left\{(\mathfrak{B}, c) \in B \mid c\left(T_{\mathfrak{B}}\right)=\right.$ inc $\}$,

- $\left(T,\left(\mathfrak{B}_{0}, c_{0}\right),\left(\mathfrak{B}_{1}, c_{1}\right), \ldots,\left(\mathfrak{B}_{k}, c_{k}\right)\right) \in \Gamma \quad$ iff $\quad T_{\mathfrak{B}_{0}}=T, \mathcal{T}_{\mathfrak{B}_{0}} \backslash\left\{T_{\mathfrak{B}_{0}}\right\}=\left\{T_{\mathfrak{B}_{1}}, \ldots, T_{\mathfrak{B}_{k}}\right\}$,

$T_{\mathfrak{B}_{0}} \in \mathcal{T}_{\mathfrak{B}_{i}}, c_{i}\left(T_{\mathfrak{B}_{i}}\right)=c_{0}\left(T_{\mathfrak{B}_{i}}\right)$, and $d_{\mathfrak{B}_{i}}\left(T_{\mathfrak{B}_{0}}\right) \leq d_{\mathfrak{B}_{0}}\left(T_{\mathfrak{B}_{i}}\right)$ for $1 \leq i \leq k$, and for all $T^{\prime} \in \mathcal{T}_{\mathfrak{B}_{i}} \backslash\left\{T_{\mathfrak{B}_{i}}\right\}$

$$
\begin{aligned}
& -c_{i}\left(T^{\prime}\right)=\text { inc iff } \quad T<_{\mathfrak{B}_{i}} T^{\prime}, \\
& -c_{i}\left(T^{\prime}\right)=\text { const iff } T^{\prime} \sim_{\mathfrak{B}_{i}} T, \\
& -c_{i}\left(T^{\prime}\right)=\text { dec iff } T^{\prime}<_{\mathfrak{B}_{i}} T .
\end{aligned}
$$

Then the decision procedure is the same as above, but with $\mathcal{A}_{T}$ replaced by $\mathcal{A}_{T}^{\prime}$.

\section{Related logics}

The operator $\leftleftarrows$ of the fragment $\mathcal{C S} \mathcal{L}$ of $\mathcal{S} \mathcal{L}$ without the absolute distance operators $\exists<a$ and $\exists \leq a$ is closely related to the 'implication' $>$ of conditional logics. According to Lewis' [18] semantics for conditionals, propositions are interpreted in a set $W$ of possible worlds which come together with orderings $\preceq_{w} \subseteq W \times W$, for $w \in W$, which can be understood as follows: $w^{\prime} \preceq_{w} w^{\prime \prime}$ if $w^{\prime}$ is more similar or closer to $w$ than $w^{\prime \prime}$. A formula $C>D$ is true at $w$ iff, for every $\preceq_{w}$-minimal $v$ with $v \models C$, we have $v \models D$. Various authors (see, for example, $[7,29]$ ) have considered the case where the relations $\preceq_{w}$ are induced by min-spaces $(\Delta, d)$ (in conditional logic, the requirement ( $\mathrm{min})$ is often called the limit assumption) by setting

$$
w^{\prime} \preceq_{w} w^{\prime \prime} \quad \text { iff } \quad d\left(w, w^{\prime}\right) \leq d\left(w, w^{\prime \prime}\right) .
$$

Under this interpretation the operators $\leftleftarrows$ and $>$ have exactly the same expressive power: for every min-model $\mathcal{I}=\left(\Delta^{\mathcal{I}}, d^{\mathcal{I}}, A_{1}^{\mathcal{I}}, A_{2}^{\mathcal{I}}, \ldots\right)$ we have

$$
\left(A_{1}>A_{2}\right)^{\mathcal{I}}=\left(\left(A_{1} \leftleftarrows\left(A_{1} \sqcap \neg A_{2}\right)\right) \sqcup \forall \neg A_{1}\right)^{\mathcal{I}}
$$

(recall that the universal modality $\forall$ is definable using $\leftleftarrows$ ) and, conversely,

$$
\left(A_{1} \leftleftarrows A_{2}\right)^{\mathcal{I}}=\left(\left(\left(A_{1} \sqcup A_{2}\right)>A_{1}\right) \sqcap\left(A_{1}>\neg A_{2}\right) \sqcap \neg\left(A_{1}>\perp\right)\right)^{\mathcal{I}} .
$$

Relations $\preceq_{w}$ induced by symmetric distance spaces have not been considered in the conditional logic literature. According to the classification of [9], our (nominal-free) logic of arbitrary min-spaces corresponds to the conditional logic of frames satisfying the normality, reflexivity, strict centering, uniformity and connectedness condition. In particular, the ExpTime upper bound for the nominal-free fragment of $\mathcal{C} \mathcal{S} \mathcal{L}$ over arbitrary min-models, was obtained before in [9] in the framework of conditional logic. 
The requirement (min) on distance spaces is crucial for the complexity results obtained for $\mathcal{S L}$ and $\mathcal{C S} \mathcal{L}$. Observe that in arbitrary metric models the operator $\square$ defined by taking $\square C=(\top \leftleftarrows \neg C)$ actually corresponds to the interior operator of the induced topology. Thus, $\mathcal{C S} \mathcal{L}$ contains the full logic $\mathbf{S} \mathbf{4}_{u}$ of topological spaces, and so can be used for spatial representation and reasoning (see, e.g., [6]). The topological aspects of $\mathcal{C S} \mathcal{L}$ will be considered elsewhere. It will also be shown that even the nominal-free fragment of $\mathcal{C S} \mathcal{L}$ is undecidable when interpreted over $\mathbb{R}, \mathbb{Q}, \mathbb{Z}$ or their finite subspaces. The proof is by reduction of the decision problem for Diophantine equations (Hilbert's 10th problem).

The integration of description logic and similarity logic presented in this paper is closely related to the well-established construction of adding concrete domains to description logics $[3,20,21,13]$. From the expressiveness point of view, the main difference between our approach and the concrete domain approach is that in our integration the domains of the similarity spaces are part of the 'abstract' domain of the description logic, whereas concrete domains are separated from the abstract domain interpreting the description logic concepts and roles. Thus, in contrast to concrete domains we can, for example, attach attributes and properties to elements of the similarity spaces (in the example from the introduction, we introduced the role has_taste_descriptor). The computational behaviour of DLs with concrete domains is in general much worse compared to the results above: even rather weak languages for the concrete domain can result in undecidable logics. Needless to say, however, that the concrete domain approach is considerably more generic and applies to various formalisms which we cannot cover.

Acknowledgements: The work on this paper was partially supported by the U.K. EPSRC research grants GR/S61966/01 and GR/S61973/01. The authors would like to thank the anonymous referees whose constructive criticism and suggestions helped us to improve the paper.

\section{References}

[1] http://www.ncbi.nlm.nih.gov/Education/BLASTinfo/.

[2] F. Baader, D. Calvanese, D. McGuinness, D. Nardi, and P. Patel-Schneider, editors. The Description Logic Handbook. Theory, Implementation and Applications. Cambridge University Press, 2003.

[3] F. Baader and P. Hanschke. A scheme for integrating concrete domains into concept languages. In Proceedings of the 12th International Joint Conference on Artificial Intelligence, IJCAI-91, pages 452-457, Sydney (Australia), 1991.

[4] F. Baader, C. Lutz, H. Sturm, and F. Wolter. Fusions of description logics and abstract description systems. Journal of Artificial Intelligence Research (JAIR), 16:1-58, 2002.

[5] A. Chagrov and M. Zakharyaschev. Modal Logic. Oxford University Press, Oxford, 1997.

[6] A. Cohn and S. Hazarika. Qualitative spatial representation and reasoning: an overview. Fundamenta Informaticae, 43:2-32, 2001.

[7] J. P. Delgrande. Preliminary considerations on the modelling of belief change operators by metric spaces. In Proceedings of NMR, pages 118-125, 2004. 
[8] E. Emerson and C. Jutla. The complexity of tree automata and logics of programs. Siam Journal of Computing, 29:132-158, 1999.

[9] N. Friedman and J. Halpern. On the complexity of conditional logics. In Proceedings of KR'94, pages 202-213, 1994.

[10] P. Gärdenfors. How to make the Semantic Web more semantic. In A.C. Varzi and L. Vieu, editors, Formal Ontology in Information Systems, pages 19-36. IOS Press, 2004.

[11] S. Ghilardi and L. Santocanale. Algebraic and model theoretic techniques for fusion decidability in modal logics. In Proceedings of LPAR 2003, volume 2850 of LNAI, pages 152-166. Springer, 2003.

[12] V. Goranko and S. Passy. Using the universal modality. Journal of Logic and Computation, 2:203-233, 1992.

[13] V. Haarslev, C. Lutz, and R. Möller. Foundations of spatioterminological reasoning with description logics. In G. Cohn, L.K. Schubert, and S.C. Shapiro, editors, Principles of Knowledge Representation and Reasoning: Proceedings of the Sixth International Conference (KR'98), pages 112-123. Morgan-Kaufmann, 1998.

[14] V. Haarslev and R. Möller. RACER system description. In R. Goré, A. Leitsch, and T. Nipkow, editors, Proceedings of the First International Joint Conference on Automated Reasoning (IJCAR'01), number 2083 in Lecture Notes in Artifical Intelligence, pages 701-705. Springer-Verlag, 2001.

[15] I. Horrocks. The Fact++ system. http://owl.man.ac.uk/factplusplus.

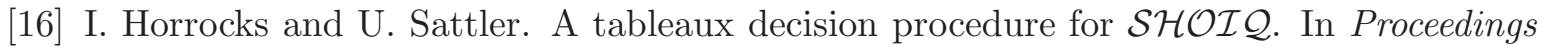
of Nineteenth International Joint Conference on Artificial Intelligence (IJCAI), pages 448-453, 2005.

[17] O. Kutz, H. Sturm, N.-Y. Suzuki, F. Wolter, and M. Zakharyaschev. Logics of metric spaces. ACM Transactions on Computational Logic, 4:260-294, 2003.

[18] D. Lewis. Counterfactuals. Blackwell, Oxford, 1973.

[19] M. Li, X. Chen, X. Li, B. Ma, and P. Vitányi. The similarity metric. In Proceedings of the Fourteenth Annual ACM-SIAM Symposium on Discrete Algorithms, pages 863-872. Society for Industrial and Applied Mathematics, 2003.

[20] C. Lutz. The Complexity of Reasoning with Concrete Domains. PhD thesis, Teaching and Research Area for Theoretical Computer Science, RWTH Aachen, 2002.

[21] C. Lutz. Description logics with concrete domains - a survey. In P. Balbiani, N.-Y. Suzuki, F. Wolter, and M. Zakharyaschev, editors, Advances in Modal Logic, volume 4, pages 265-296. King's College Publications, 2003.

[22] C. Lutz, F. Wolter, and M. Zakharyaschev. A tableau algorithm for reasoning about concepts and similarity. In M. C. Mayer and F. Pirri, editors, Automated Reasoning with Analytic Tableaux and Related Methods, volume 2796 of LNCS, pages 134-149. Springer, 2003. 
[23] B. Mirkin. Mathematical Classification and Clustering. Kluwer Academic Publishers, 1996.

[24] B. Parsia and E. Sirin. Pellet: An OWL DL-reasoner. In Proceedings of the International Workshop on Description Logics, 2004.

[25] V. Pratt. Models of program logic. In Proceedings of the 20th Annual Symposion on the Foundations of Computer Science, pages 115-122, 1979.

[26] I. Pratt-Hartmann. Complexity of the two-variable fragment with counting quantifiers. Journal of Logic, Language and Information, 14:369-395, 2005.

[27] Ph. Resnik. Semantic similarity in a taxonomy: An information-based measure and its application to problems of ambiguity in natural language. Journal of Artificial Intelligence Research (JAIR), 11:95-130, 1999.

[28] W. Sayers. An Introduction to Library Classification. Grafton \& Co, London, 1955.

[29] K. Schlechta. Coherent Systems. Elsevier, 2004.

[30] M. Sheremet, D. Tishkovsky, F. Wolter, and M. Zakharyaschev. Comparative similarity, tree automata, and Diophantine equations. In Proceedings of LPAR 2005, volume 3835 of LNAI, pages 651-665. Springer, 2005.

[31] M. Sheremet, D. Tishkovsky, F. Wolter, and M. Zakharyaschev. From topology to metric: modal logic and quantification in metric spaces. In G. Governatori, I. Hodkinson, and Y. Venema, editors, Advances in Modal Logic, volume 6, pages 429-448. College Publications, 2006.

[32] E. Spaan. Complexity of Modal Logics. PhD thesis, University of Amsterdam, 1993.

[33] W. Thomas. Automata on infinite objects. In Handbook of Theoretical Computer Science, volume B, pages 133-191. Elsevier, 1990.

[34] S. Tobies. The complexity of reasoning with cardinality restrictions and nominals in expressive description logics. Journal of Artificial Intelligence Research (JAIR), 12:199217, May 2000.

[35] A. Tversky. Features of similarity. Psychological Review, 84:327-352, 1977.

[36] M. Vardi and P. Wolper. Automata-theoretic techniques for modal logics of programs. Journal of Computer and System Sciences, 32:183-221, 1986.

[37] D. Wishart. Whisky Classified. Choosing single malts by flavour. Pavilion Books, London, 2002.

[38] F. Wolter and M. Zakharyaschev. Reasoning about distances. In Proceedings of the 18th International Joint Conference on Artificial Intelligence (IJCAI 2003), pages 1275-1280. Morgan Kaufmann, 2003.

[39] F. Wolter and M. Zakharyaschev. A logic for metric and topology. Journal of Symbolic Logic, 70:795-828, 2005. 
[40] Y. Zhou, J. Young, A. Santrosyan, K. Chen, S. Yan, and E. Winzeler. In silico gene function prediction using ontology-based pattern identification. Bioinformatics, 21:1237$1245,2005$. 